\title{
Robots Driven by Compliant Actuators: Optimal Control Under Actuation Constraints
}

\author{
David J. Braun, Member, IEEE, Florian Petit, Felix Huber, Sami Haddadin, Member, IEEE, Patrick van der Smagt, \\ Alin Albu-Schäffer, Member, IEEE, and Sethu Vijayakumar
}

\begin{abstract}
Anthropomorphic robots that aim to approach human performance agility and efficiency are typically highly redundant not only in their kinematics but also in actuation. Variableimpedance actuators, used to drive many of these devices, are capable of modulating torque and impedance (stiffness and/or damping) simultaneously, continuously, and independently. These actuators are, however, nonlinear and assert numerous constraints, e.g., range, rate, and effort limits on the dynamics. Finding a control strategy that makes use of the intrinsic dynamics and capacity of compliant actuators for such redundant, nonlinear, and constrained systems is nontrivial. In this study, we propose a framework for optimization of torque and impedance profiles in order to maximize task performance, which is tuned to the complex hardware and incorporating real-world actuation constraints. Simulation study and hardware experiments 1) demonstrate the effects of actuation constraints during impedance control, 2) show applicability of the present framework to simultaneous torque and temporal stiffness optimization under constraints that are imposed by real-world actuators, and 3) validate the benefits of the proposed approach under experimental conditions.
\end{abstract}

Index Terms-Dynamics, optimal impedance control, redundant robots, variable impedance actuation.

\section{INTRODUCTION}

$\mathbf{M}$ ODERN anthropomorphic robots, which aim to approach human behavior and performance, are highly

Manuscript received August 27, 2012; revised February 12, 2013; accepted June 18, 2013. Date of publication July 29, 2013; date of current version September 30, 2013. This paper was recommended for publication by Associate Editor N. Simaan and Editor G. Oriolo upon evaluation of the reviewers' comments. This work was supported by the European Commission's Seventh Framework Programmes as part of the STIFF (grant number 231576) and VIACTORS (grant number 231554) projects and in part by the Sixth Framework Programme as part of the SAPHARI (grant number 287513) project. The authors also acknowledge the support of the DLR Hand-Arm System design team. This paper was presented in part at the IEEE/RSJ International Conference on Intelligent Robotic Systems, Algarve, Portugal, October 2012.

D. J. Braun and S. Vijayakumar are with the School of Informatics, University of Edinburgh, Edinburgh EH8 9AB, U.K. (e-mail: david.braun@ed.ac.uk; sethu.vijayakumar@ed.ac.uk).

F. Petit, F. Huber, S. Haddadin, and A. Albu-Schäffer are with the German Aerospace Centre, Institute of Robotics and Mechatronics, OberpfaffenhofenWeßling, Oberpfaffenhofen, Wessling 82234, Germany (e-mail: Florian.Petit@ dlr.de; f.huber@dlr.de; Sami.Haddadin@dlr.de; Alin.Albu-Schaeffer@dlr.de).

P. van der Smagt is with the Institute for Informatics, Technische Universität, München 80333, Germany (e-mail: smagt@brml.org).

This paper has supplementary downloadable video available at $\mathrm{http}: / /$ ieeexplore.ieee.org provided by the authors. It demonstrates the proposed framework implemented on two conceptually different variable stiffness devices. The material is $48 \mathrm{MB}$ in size.

Color versions of one or more of the figures in this paper are available online at http://ieeexplore.ieee.org.

Digital Object Identifier 10.1109/TRO.2013.2271099

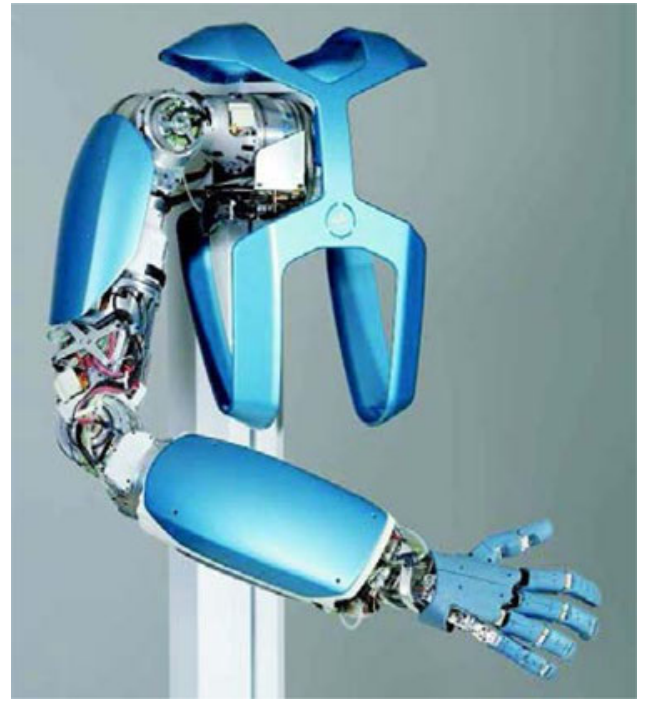

Fig. 1. DLR Hand-Arm System resembles the complexity of a human upper limb. This device has 26 kinematic degrees of freedom, all driven by variable stiffness actuators. These actuators incorporate 52 motors.

redundant, not only in their kinematics but in their actuation as well (e.g., DLR Hand-Arm System [1]; see Fig. 1). Variable impedance actuators (VIAs) [2]-[9], used to drive many of these devices, are capable of simultaneous torque and passive impedance (i.e., stiffness and/or damping) modulation as opposed to more classic actuators on torque controlled robots [10]. The premise of impedance control [11] and the variable impedance actuation paradigm is to increase the performance i.e., dynamic range, agility, and safety of robots [12]-[17]. This, however, does not come for free. Indeed, VIAs introduce nonlinearities by design, employ multiple motors per joint, and assert important actuation constraints such as range, rate, and effort limitations, that are often neglected on classical devices. Due to these reasons, the potential that is offered by variable impedance actuation may only be exploited if the control problem at hand is algorithmically treated and if the control redundancy is optimally resolved in a task specific manner [18]. However, there are number of challenges to this:

1) First, it is known that the model structure of a compliantly actuated robot is more complex than that of rigid robots. As such, it is often nontrivial to find the right representation of the dynamics that is neither too complex to be computationally intractable nor too simple to lack validity with respect to the real physics of the system.

One of the widely used models of elastic-joint robots, with constant joint stiffness, was derived by Spong [19]. In this 
model, the tradeoff between complexity and physical validity was balanced by neglecting the inertial coupling between the motor and the link-side dynamics. This was shown to be viable under highly geared actuation. If this design condition is not provided, one may be required to work with a more general model, as the one introduced by Tomei [20]. There is considerable interest in providing extensions for such models for systems with variable joint elasticity. To this end, there are various formulations that are derived using Spong's assumption and by assuming "arbitrarily fast" dynamics of stiffness modulation.

In Section II, we propose a general model for compliantly actuated robots driven with series-elastic and variable-stiffness actuators (i.e., SEAs [21] and VSAs [8]) that: 1) incorporates the inertial coupling between the rigid body and the motor dynamics; 2) is valid for (physically and/or geometrically) nonlinear, redundantly actuated systems, and 3) in the case of VSAs, includes the real dynamics that are associated with stiffness modulation. As derived, this model generalizes those frequently used for elastic joint robots, while it also extends representations of variable stiffness systems recently presented in the literature [22]-[24]. Based on this model, we propose a physically consistent minimalistic representation of the dynamics for optimal control planning. In Section VII, this representation is tested (and shown to be viable) for complex variable stiffness robots that perform dynamic movements.

2) Second, it is of interest to find a suitable control strategy that enables exploitation of the natural dynamics of the system through utilization of the intrinsic compliance of the actuators.

The control literature of elastic joint robots that have constant joint stiffness contains various approaches, especially to the position control problem [25]. Among these are the singular perturbation-based approaches [26], decoupling-based approaches [19], [27], backstepping-based schemes [28], and the passivity-based approaches [29], [30] to mention a few. In the same context, combined position and stiffness control of variable stiffness robots is more recently investigated using (a decoupling-based) feedback linearizion [31], [32]. These approaches generally combine closed-loop motor position and velocity control with link-side feedback on positions, torques, and their derivatives. Using the former, one compensates the uncertainties in the motor dynamics, while using the latter, one may track desired link-side position (and stiffness) trajectories.

In this paper, we assume that the model of the system dynamics is reasonably well identified: either using standard system identification [33] or online learning [34], [35]. Under this assumption, we investigate an approach to open-loop elastic torque and stiffness modulation. In this formulation (see Sections III and IV), the link-side motion is not preplanned and tracked in a traditional sense, but instead it is a consequence of the mechanical properties of the link-side dynamics and the output of the compliant actuators. This output is provided by the elastic torques and stiffness that is continuously modulated through an optimally planned motor program. This motor program is implemented through motor position and velocity feedback. Accordingly, we employ active feed-back control to gain robustness with respect to uncertainties in the motor dynam- ics, while relying on the mechanical feedback at the link-side dynamics that is provided by the physical compliance of the actuators. ${ }^{1}$ It is important to note that using VIAs, this physical compliance is not fixed but can be optimally modulated during the motion. This allows one to fit the intrinsic properties of the system to the task requirement, that in turn enables better exploitation of the system dynamics during the movement.

3) Third, it is vital to employ algorithmic methods that can, in a unified way, treat the nonlinear coupling between motion, torque, and impedance characteristics inherent to systems driven with compliant actuators, and that allow planning impedance control strategies under real-world actuation constraints.

One way to define the desired impedance is through the optimal control formulation, where the control task is encoded by an objective functional [37]. Using this idea, it was demonstrated how impedance adaptation can decrease the energy that is required for movement generation [38] and how impedance optimization can be used to enhance safety in human-robot interactions [14]. Along this line of research, the benefit of temporal impedance optimization, and accordingly impedance modulation during movement, has also been recently investigated [16], [17], [24], [39]-[41]. In this context, using algorithmic tools was recognized as especially important since VIAs introduce nonlinear coupling between motion, torque, and impedance characteristics, actuation redundancy (increased dimensionality of the control inputs), and actuation constraints (range, rate, acceleration limits introduced in Section V). These make heuristic methods less effective when used in planning and control on compliantly actuated systems.

In Section VI, we consider planning optimal impedance control strategies under actuation constraints [42], [43]. While analytical approaches may have limitations for constrained problems, there are numerical methods that can be effective in this context [44], [45]. To this end, we provide a systematic treatment of constraints on robotic systems by explicitly embedding nonlinear state inequality constraints into the dynamics. In this way, the number of state constraints may either be reduced or even fully eliminated from the consideration. As a result, state constraints, that would otherwise require problem specific derivation and sophisticated computation, become easier to treat numerically. In Section VII, we demonstrate the viability of this framework in simulations and hardware experiments. The material that is presented in the last two sections is an extended exposition of our previous work [24].

In summary, we present a model-based constrained optimal control framework, realizing (variable) impedance control tuned to the task, and specificity of the system dynamics. The promise of this framework is: applicability to complex compliantly actuated robots that perform dynamic tasks under real-world conditions.

\footnotetext{
${ }^{1}$ This is unlike active stiffness control (e.g., [36]) realized through link-side position feedback on torque controllable devices.
} 


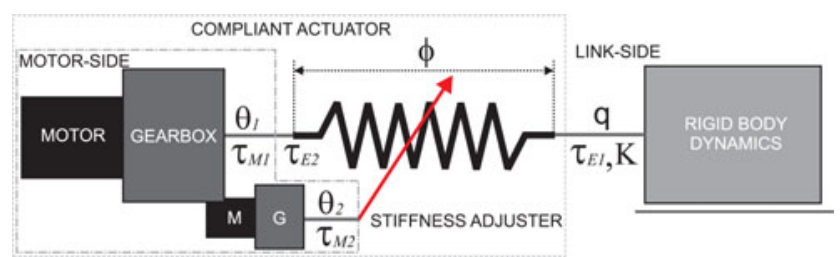

Fig. 2. Schematic representation of a compliantly actuated robot: $\boldsymbol{\theta}$ (motor positions), $\boldsymbol{\tau}_{M}$ (motor torques), $\boldsymbol{\phi}$ (spring length), $\boldsymbol{\tau}_{E 2}$ (motor-side elastic torques), $\boldsymbol{\tau}_{E 1}$ (link-side elastic torques), $\mathbf{K}$ (link-side stiffness of the actuators) q (link positions).

\section{Model of a Compliantly Actuated Robot}

In this section, we present a dynamic model for compliantly actuated robotic systems. In this model, the classical rigid body dynamics of the robot is supplemented with the model of the compliant actuators. These compliant actuators can be SEA [21] or VSA [8] with no restriction on generality.

\section{A. Robot Dynamics}

Consider an $n$-DOF autonomous robotic system, the configuration of which is uniquely specified by $\mathbf{q} \in \mathbb{R}^{n}$ generalized coordinates (e.g., joint angles). Let this system be equipped with $m$ compliant actuators (e.g., SEAs and/or VSAs), and let $\boldsymbol{\theta} \in \mathbb{R}^{m}(m \geq n)$ denote the motor angles reflected through gear reduction (see Fig. 2). In the remainder of this paper, we refer to $\mathbf{q}$ and $\boldsymbol{\theta}$ as link-side and motor-side coordinates, respectively. Due to standard geometric features, we assume that:

A.1: the rotation axes of the motors and gearboxes are axes of symmetry and correspondingly they coincide with the principal axes of inertia. $^{2}$

Based on (A.1), we next define the kinetic and the gravitational potential energy of the rigid body dynamics and the dynamics of the motors. In addition, we also define the elastic potential energy to characterize the elastic torques/forces that are generated by the actuators. Using these energy functions, the equation of motion of the compliantly actuated system is derived through application of the Lagrangian formalism [47].

1) Kinetic Energy: Under assumption (A.1), the kinetic energy of the considered dynamical system reads

$$
\mathcal{T}=\underbrace{\frac{1}{2} \dot{\mathbf{q}}^{T} \mathbf{M}(\mathbf{q}) \dot{\mathbf{q}}}_{\mathcal{T}_{11}(\mathbf{q}, \dot{\mathbf{q}})}+\underbrace{\dot{\mathbf{q}}^{T} \mathbf{S}(\mathbf{q}) \dot{\boldsymbol{\theta}}}_{\mathcal{T}_{12}(\mathbf{q}, \dot{\mathbf{q}}, \dot{\boldsymbol{\theta}})}+\underbrace{\frac{1}{2} \dot{\boldsymbol{\theta}}^{T} \mathbf{J} \dot{\boldsymbol{\theta}}}_{\mathcal{T}_{22}(\dot{\boldsymbol{\theta}})}
$$

where $\mathbf{M} \in \mathbb{R}^{n \times n}$ is the symmetric and positive definite inertia matrix of the rigid body dynamics (that may in general include the inertial parameters of the actuators), $\mathbf{S} \in \mathbb{R}^{n \times m}$ represents the inertial coupling between the rigid body and the motor dynamics, while $\mathbf{J} \in \mathbb{R}^{m \times m}$ is a constant and diagonal matrix that contains the inertia of the motors/gearboxes. In the aforementioned relation, the inertial coupling between the link-side and the motor-side dynamics is represented by $\mathcal{T}_{12}$. This term exists since the rotational kinetic energy of each motor/gearbox

\footnotetext{
${ }^{2}$ This is a standard assumption for models of elastic-joint robots, see [19], [20], [46] (assumption A.2 in these references).
}

assembly is not only due to their self-rotation, but also due to the rotation of the actuators induced by the motion of the robot.

2) Gravitational Potential Energy: Under the assumption (A.1), the center of the mass of the actuators will not change due to the self-rotation of the rotors and the gears. This means practically that the center of mass of the composed system does not depend on $\boldsymbol{\theta}$. Direct implication of this is that the gravitational potential energy of the entire system only depends on the configuration of the rigid body dynamics

$$
\mathcal{U}_{G}=\mathcal{U}_{G}(\mathbf{q})
$$

3) Elastic Potential Energy: In this paper, we consider series elastic and variable stiffness actuators where:

\section{A.2: the actuator compliance is provided by elastic elements}

(i.e., linear and/or nonlinear springs).

Under this (fairly general) assumption, the conservative elastic forces by the actuators can be characterized with a potential energy function ${ }^{3}$

$$
\mathcal{U}_{E}=\mathcal{U}_{E}(\mathbf{q}, \boldsymbol{\theta})=-\sum_{i=1}^{p} \int_{l_{i 0}}^{l_{i}(\mathbf{q}, \boldsymbol{\theta})} F_{i}(s) d s
$$

where $l_{i}$ and $l_{i 0}$ denote the length and equilibrium length of the $i$ th spring, while $F_{i}=F_{i}\left(l_{i}\right)$ is the corresponding spring force $F_{i}\left(l_{i 0}\right)=0$. In the following, we employ (3) to define the elastic torques that are provided by the compliant actuators.

4) Equation of Motion: Using the Lagrangian formalism, the equation of motion of the robotic system becomes

$$
\begin{aligned}
{\left[\begin{array}{cc}
\mathbf{M} & \mathbf{S} \\
\mathbf{S}^{T} & \mathbf{J}
\end{array}\right]\left[\begin{array}{c}
\ddot{\mathrm{q}} \\
\ddot{\boldsymbol{\theta}}
\end{array}\right] } & +\left[\begin{array}{cc}
\mathbf{C} & \mathbf{C}_{q} \\
\mathbf{C}_{\theta} & \mathbf{0}
\end{array}\right]\left[\begin{array}{c}
\dot{\mathbf{q}} \\
\dot{\boldsymbol{\theta}}
\end{array}\right]+\left[\begin{array}{c}
\mathbf{D} \dot{\mathbf{q}} \\
\mathbf{D}_{\theta} \dot{\boldsymbol{\theta}}
\end{array}\right] \\
& +\left[\begin{array}{c}
\mathbf{G} \\
\mathbf{0}
\end{array}\right]=\left[\begin{array}{c}
\boldsymbol{\tau}_{E 1} \\
\boldsymbol{\tau}_{E 2}
\end{array}\right]+\left[\begin{array}{c}
\mathbf{0} \\
\boldsymbol{\tau}_{M}
\end{array}\right]
\end{aligned}
$$

where the inertia matrix is defined by $\mathbf{M}=\partial \mathcal{T}^{2} / \partial \dot{\mathbf{q}}^{2}, \mathbf{S}=$ $\partial \mathcal{T}^{2} / \partial \dot{\mathbf{q}} \partial \dot{\boldsymbol{\theta}}$, and $\mathbf{J}=\partial \mathcal{T}^{2} / \partial \dot{\boldsymbol{\theta}}^{2}$, while

$$
\begin{aligned}
\mathbf{C} \dot{\mathbf{q}} & =\mathbf{C}(\mathbf{q}, \dot{\mathbf{q}}, \dot{\boldsymbol{\theta}}) \dot{\mathbf{q}}=\frac{\partial^{2}\left(\mathcal{T}_{11}+\mathcal{T}_{12}\right)}{\partial \dot{\mathbf{q}} \partial \mathbf{q}} \dot{\mathbf{q}}-\frac{\partial \mathcal{T}_{11}}{\partial \mathbf{q}} \\
\mathbf{C}_{q} \dot{\boldsymbol{\theta}} & =\mathbf{C}_{q}(\mathbf{q}, \dot{\mathbf{q}}) \dot{\boldsymbol{\theta}}=-\frac{\partial \mathcal{T}_{12}}{\partial \mathbf{q}} \\
\mathbf{C}_{\theta} \dot{\mathbf{q}} & =\mathbf{C}_{\theta}(\mathbf{q}, \dot{\mathbf{q}}) \dot{\mathbf{q}}=\frac{\partial^{2} \mathcal{T}_{12}}{\partial \dot{\boldsymbol{\theta}} \partial \mathbf{q}} \dot{\mathbf{q}} \\
\mathbf{G} & =\mathbf{G}(\mathbf{q})=\frac{\partial \mathcal{U}_{G}}{\partial \mathbf{q}} \\
\boldsymbol{\tau}_{E 1} & =\boldsymbol{\tau}_{E 1}(\mathbf{q}, \boldsymbol{\theta})=-\frac{\partial \mathcal{U}_{E}}{\partial \mathbf{q}}
\end{aligned}
$$

\footnotetext{
${ }^{3}$ If the elastic elements are linear springs, $F_{i}=k_{i}\left(l_{i}-l_{i 0}\right)$, then (3) simplifies to

$$
\mathcal{U}_{E}(\mathbf{q}, \boldsymbol{\theta})=\frac{1}{2}\left(\mathbf{l}(\mathbf{q}, \boldsymbol{\theta})-\mathbf{l}_{0}\right)^{T} \mathbf{K}_{s}\left(\mathbf{l}(\mathbf{q}, \boldsymbol{\theta})-\mathbf{l}_{0}\right)
$$

where $\mathbf{l}(\mathbf{q}, \boldsymbol{\theta})=\left[l_{1}, l_{2}, \ldots, l_{p}\right]^{T}$ is the length of the springs, $\mathbf{l}_{\mathbf{0}}=\left[l_{01}, l_{02}\right.$, $\left.\ldots, l_{0 p}\right]^{T}$ are the equilibrium lengths, while $\mathbf{K}_{s}=\operatorname{diag}\left\{\left[k_{s 1}, k_{s 2}, \ldots, k_{s p}\right]\right\}$ is a diagonal matrix that contains the stiffness constants of the springs.
} 


$$
\boldsymbol{\tau}_{E 2}=\boldsymbol{\tau}_{E 2}(\mathbf{q}, \boldsymbol{\theta})=-\frac{\partial \mathcal{U}_{E}}{\partial \boldsymbol{\theta}}
$$

where $\mathbf{C} \in \mathbb{R}^{n \times n}, \mathbf{C}_{q} \in \mathbb{R}^{n \times m}$, and $\mathbf{C}_{\theta} \in \mathbb{R}^{m \times n}$ are matrices that represent the Coriolis and normal inertial forces, Dq $\in \mathbb{R}^{n}$ and $\mathbf{D}_{\theta} \dot{\boldsymbol{\theta}} \in \mathbb{R}^{m}$ are the forces due to viscous friction (may be derived using the virtual work principle), $\mathbf{G} \in \mathbb{R}^{n}$ are the gravitational forces, $\tau_{E 1} \in \mathbb{R}^{n}$ are the elastic joint torques that affect the rigid body dynamics, $\tau_{E 2} \in \mathbb{R}^{m}$ contains the elastic reaction torques, at the input to the actuators, that affects the motor dynamics, while $\tau_{M} \in \mathbb{R}^{m}$ are the motor torques. Accordingly, the first term in (4) represents the link-side dynamics, while the second term corresponds to the motor-side dynamics.

As derived, equation (4) provides a general model of a compliantly actuated robot. In particular, it extends the model of elastic joint robots that are proposed in [20], since it remains valid for redundantly actuated systems (i.e., $m \geq n$ ), and is applicable to robots that have nonlinear and variable joint elasticity. Compared with the model that has been proposed for variable stiffness robots in [24], (4) accommodates the inertial coupling between the motors and the link-side dynamics (and as such it remains valid even if the motor gearing is not particularly high). Compared with the model that has been proposed in [23], (4) is derived for compliant actuators with no assumption on the physical/geometric nonlinearities they introduce to the system.

\section{ON Motor Position CONTROL OF COMPLiAntLY ACTUATED SYSTEMS}

In this paper, we investigate an approach to feed-forward elastic torque and stiffness modulation. In this approach, the elastic joint torques given by (9) and the associated joint stiffness (defined in Section IV-B) are optimally modulated through a motor program. This motor program is defined by the desired motor trajectories that are implemented through closed-loop control introduced next.

\section{A. Closed-Loop Motor Dynamics}

According to the full model (4), (7), and (10), the actuator dynamics is defined by

$$
\mathbf{J} \ddot{\boldsymbol{\theta}}=\boldsymbol{\tau}_{M} \underbrace{-\mathbf{S}^{T}(\mathbf{q}) \ddot{\mathbf{q}}-\mathbf{C}_{\theta}(\mathbf{q}, \dot{\mathbf{q}}) \dot{\mathbf{q}}-\mathbf{D}_{\theta} \dot{\boldsymbol{\theta}}+\boldsymbol{\tau}_{E 2}(\mathbf{q}, \boldsymbol{\theta})}_{\boldsymbol{\tau}_{\theta}(\mathbf{q}, \dot{\mathbf{q}}, \ddot{\mathbf{q}}, \boldsymbol{\theta})}
$$

where $\mathbf{J}=\mathbf{n}^{2} \mathbf{J}_{m}, \mathbf{J}_{m} \in \mathbb{R}^{m \times m}$ is a diagonal matrix that contains the rotational inertia of the motors and gearboxes (at the input to the gear reducers), $i \in\{1,2, \ldots, m\}, \mathbf{n}^{2}=\operatorname{diag}\left\{n_{i}^{2}\right\} \in$ $\mathbb{R}^{m \times m}$ are the squared gear ratios, while $\boldsymbol{\tau}_{\theta} \in \mathbb{R}^{m}$ denotes the inertial effects from the link dynamics and the reaction torques by the compliant elements that affect the actuators.

In order to perform motor position control, we employ the following control law

$$
\boldsymbol{\tau}_{M}=-\hat{\boldsymbol{\tau}}_{\theta}-\mathbf{n}^{2} \mathbf{K}_{m}\left(\boldsymbol{\theta}-\boldsymbol{\theta}_{d}\right)-\mathbf{n}^{2} \mathbf{B}_{m} \dot{\boldsymbol{\theta}}
$$

where the feed-forward component $\hat{\boldsymbol{\tau}}_{\theta}$ is the estimate of $\boldsymbol{\tau}_{\theta}$ in (11), $\mathbf{K}_{m}=\operatorname{diag}\left\{k_{m i}\right\} \in \mathbb{R}^{m \times m}$, and $\mathbf{B}_{m}=\operatorname{diag}\left\{b_{m i}\right\} \in$ $\mathbb{R}^{m \times m}$ are the user-defined (servo) gains while $\boldsymbol{\theta}_{d}=\boldsymbol{\theta}_{d}(t) \in$
$\mathbb{R}^{m}$ is the desired motor position. By substituting (12) into (11), the closed-loop motor dynamics becomes

$$
\ddot{\boldsymbol{\theta}}+2 \boldsymbol{\beta} \dot{\boldsymbol{\theta}}+\boldsymbol{\kappa}^{2} \boldsymbol{\theta}=\boldsymbol{\kappa}^{2} \boldsymbol{\theta}_{e}
$$

where $\boldsymbol{\beta}=\frac{1}{2} \mathbf{J}_{m}^{-1} \mathbf{B}_{m}, \boldsymbol{\kappa}=\mathbf{J}_{m}^{-1} \mathbf{K}_{m}$, while $\boldsymbol{\theta}_{e}$ is the motor equilibrium position defined by

$$
\boldsymbol{\theta}_{e}=\boldsymbol{\theta}_{d}+\left(\mathbf{n}^{2} \mathbf{K}_{m}\right)^{-1} \Delta \boldsymbol{\tau}_{\theta}
$$

and $\Delta \boldsymbol{\tau}_{\theta}=\boldsymbol{\tau}_{\theta}-\hat{\boldsymbol{\tau}}_{\theta}$. Following the aforementioned relation, we can define three conditions under which the actuator dynamics may not be (significantly) affected by link-side motion [i.e., state dependent torques included in $\tau_{\theta}$, see (11)]. Indeed, if

C.1 the inertial torques and the elastic reaction torques are well identified: $\hat{\boldsymbol{\tau}}_{\theta} \approx \boldsymbol{\tau}_{\theta} \Rightarrow \Delta \boldsymbol{\tau}_{\theta} \approx \mathbf{0}$

C.2 the motors operate through high gear reduction: $\min \left\{n_{1}, n_{2}, \ldots, n_{m}\right\} \gg 1$;

C.3 the control torques (12) are realized with high position gains: $\min \left\{k_{m 1}, k_{m 2}, \ldots, k_{m m}\right\} \gg 1$;

then the last term in (14) becomes negligible i.e., $\boldsymbol{\theta}_{e} \approx \boldsymbol{\theta}_{d}(t)$ and the actuator model, (13) and (14), simplifies to

$$
\ddot{\boldsymbol{\theta}}+2 \boldsymbol{\beta} \dot{\boldsymbol{\theta}}+\boldsymbol{\kappa}^{2} \boldsymbol{\theta}=\boldsymbol{\kappa}^{2} \boldsymbol{\theta}_{d} .
$$

The first condition (C.1) requires precise system identification of the motor-side dynamics that may or may not be provided. On the other hand, high gear reduction (C.2) is often used on robotic systems, and a high position gain (C.3) is a standard attribute of any servo-control system. Accordingly, we assume that at least one of the latter two conditions is satisfied, and as such (15) is a valid representation of the closed-loop motor dynamics. $^{4}$

Regarding the choice of the parameters in (15) we note that: 1) critical damping, i.e., $\boldsymbol{\beta}=\boldsymbol{\kappa}$ leads to the fastest response without overshoot; and 2) servo-control, i.e., $\forall \kappa_{i} \gg 1$, leads to $\boldsymbol{\theta} \approx \boldsymbol{\theta}_{d}$ independent of the specificity of the motor side dynamics (11). In Sections VI-A and VI-E, we will invoke both of these arguments to ensure that the constraint consistent motor program $\boldsymbol{\theta}_{d}$ leads to optimal constraint consistent motor trajectories $\boldsymbol{\theta}$, irrespective of the motor-side dynamics (11).

It is of interest to note at this point that highly geared motor units (C.2) and/or servo-control (C.3) do not cancel out the intrinsic link-side dynamics of the robot on compliantly actuated systems. This is because the compliance of the (SE and VS) actuators effectively decouples the link-side from the motorside dynamics. This is in contrast with rigid actuators, where bandwidth limitations on the servo-controlled motor dynamics directly limits the speed/acceleration of the link-side motion. Moreover, this decoupling is the reason why identification of the link-side dynamics, i.e., the first equation in (4), is easier on compliantly actuated systems than on their rigidly actuated counterparts. ${ }^{5}$ This makes adequate system identification and

\footnotetext{
${ }^{4}$ This argument holds (under C.2 and/or C.3) even if no model-specific feedforward torque is included in the control law (12) (i.e., $\hat{\boldsymbol{\tau}}_{\theta} \equiv \mathbf{0}$ ).

${ }^{5}$ This is because the nonlinear (and possibly nonsmooth) frictional terms, that are difficult to model and identify, are decoupled from the link-side dynamics by the intrinsic compliance of the actuators.
} 
correspondingly feed-forward elastic torque/stiffness modulation viable on series elastic and variable impedance systems e.g., [18], [24].

\section{Model of a COMPliant ACTUATOR}

In this section, we propose a general representation of compliant, series elastic, and variable stiffness actuators, where the motor positions are the control inputs while the elastic joint torque and the joint stiffness are the actuators output. Here we: 1) define the torque and stiffness characteristics of these actuators; 2) identify the necessary and sufficient conditions that make independent torque and stiffness modulation viable; and 3 ) introduce the fundamental smoothness conditions inherent to compliant actuated systems.

\section{A. Torque Characteristic of the Actuators}

According to (9), using the motor side positions $\boldsymbol{\theta}$ as inputs

$$
\boldsymbol{\tau}_{E 1}=\boldsymbol{\tau}_{E 1}(\mathbf{q}, \boldsymbol{\theta})
$$

provides the elastic joint torques as actuator outputs. ${ }^{6}$ The state (i.e., q) dependence of this static torque characteristic is due to the passive elasticity, built into the actuators, and not due to active feedback [36]. By setting a constant motor position $\boldsymbol{\theta}=\mathbf{c},(16)$ defines the passive torque characteristic of the actuators, $\boldsymbol{\tau}_{c}(\mathbf{q})=\boldsymbol{\tau}(\mathbf{q}, \mathbf{c})$, while by changing the motor positions, $\boldsymbol{\theta}=\boldsymbol{\theta}(t)$, these passive mechanical characteristic are actively modulated. In order to do this, however, the actuators should be torque controllable. A necessary condition for torque controllability is provided if the number of motor inputs is at least equal to the number of torque outputs (i.e., $m \geq n$ ), while a sufficient condition is given by

$$
d=\operatorname{det}\left(\left[\frac{\partial \boldsymbol{\tau}}{\partial \boldsymbol{\theta}}\right]\left[\frac{\partial \boldsymbol{\tau}}{\partial \boldsymbol{\theta}}\right]^{+}\right) \neq 0 .
$$

It may be of interest to note that this condition is state dependent, and that real VSAs may have singular regions in their work space, where torque controllability is not provided. While this can lead to reduced control authority, it may not adversely affect the present feed-forward scheme since we do not use the inversion of (16) in the implementation.

\section{B. Stiffness Characteristic of the Actuators}

By definition, stiffness relates the torque response to position perturbation. In this light, the output stiffness of the actuators $\mathbf{K} \in \mathbb{R}^{n \times n}$, that is, the stiffness of the actuators seen by the rigid body dynamics, is given by

$$
\mathbf{K}=\mathbf{K}(\mathbf{q}, \boldsymbol{\theta})=-\frac{\partial \tau}{\partial \mathbf{q}} .
$$

By analogy with the previous section, one can define the design specific (often highly non-linear) passive stiffness characteristics of the actuators by fixing the motor positions,

\footnotetext{
${ }^{6}$ For notational convenience, we drop the index in the aforementioned equation, such that $\boldsymbol{\tau}=\boldsymbol{\tau}_{E 1}$ will denote the elastic joint torques in the remainder of this paper.
}

$\mathbf{K}_{c}(\mathbf{q})=\mathbf{K}(\mathbf{q}, \mathbf{c})$. On the other hand, using time-dependent motor positions, the actuator stiffness and torque can be simultaneously modulated, although they may not be independent. Independent modulation of $\boldsymbol{\tau}(\mathbf{q}, \boldsymbol{\theta})$ and $\mathbf{K}(\mathbf{q}, \boldsymbol{\theta})$ through $\boldsymbol{\theta}(t)$ $i$, however, one of the key attributes of variable-stiffness actuators. The necessary condition to achieve that is provided by actuation redundancy (i.e., $m>n$ ), while a sufficient condition is given by

$$
r=\operatorname{rank}\left(\left[\frac{\partial \mathbf{k}}{\partial \boldsymbol{\theta}}\right]\left(\mathbf{I}-\left[\frac{\partial \boldsymbol{\tau}}{\partial \boldsymbol{\theta}}\right]^{+}\left[\frac{\partial \boldsymbol{\tau}}{\partial \boldsymbol{\theta}}\right]\right)\right) \neq 0
$$

where $\mathbf{k}=\operatorname{vec}(\mathbf{K}) \in \mathbb{R}^{n^{2}}$ is the column vectorized stiffness matrix, $\mathbf{I} \in \mathbb{R}^{m \times m}$ is an identity matrix, while $(*)^{+}$denotes the Moore-Penrose generalized inverse of $(*)$ (see [48]).

Practically, (19) ensures that the motor positions that cannot affect the joint torques, can be used to modulate the joint stiffness. It is important to note that (19) is not sufficient to modulate all components of the stiffness matrix. Indeed, the number of components that can be independently modulated through $\boldsymbol{\theta}$ is defined by $r$. It is also important to recognize that the above condition is not satisfied for series elastic actuators (i.e., $\left.m=n \Rightarrow \mathbf{I}-(\partial \boldsymbol{\tau} / \boldsymbol{\theta})^{+}(\partial \boldsymbol{\tau} / \boldsymbol{\theta}) \equiv \mathbf{0}\right)$, while it must be satisfied, at least for some portion of the work space, for variable stiffness actuators.

\section{Fundamental Smoothness Conditions}

When the smoothness properties of the link and the motorside positions, ${ }^{7}$ i.e., $\boldsymbol{\theta}(t) \in \mathbb{C}^{1}$ and $\mathbf{q}(t) \in \mathbb{C}^{2}$ are reflected through the actuators, ${ }^{8}$ the output torque and stiffness characteristics admit the following continuity and smoothness conditions as functions of time:

$$
\boldsymbol{\tau}(\mathbf{q}(t), \boldsymbol{\theta}(t)) \in \mathbb{C}^{1} \text { and } \mathbf{K}(\mathbf{q}(t), \boldsymbol{\theta}(t)) \in \mathbb{C}^{0} .
$$

The previous two conditions may be seen as fundamental smoothness requirements that are asserted by general principle of dynamics to a general class of compliant actuators that admit continuously differentiable torque-angle characteristic ${ }^{9}$ (16).

It is of interest to note here that exact link-side position and stiffness tracking on variable stiffness systems is only possible if the desired position/stiffness trajectories are at least four/two times differentiable, respectively (see [32]). Compared with (20), such a tracking scheme requires stronger conditions, i.e., an actuator that provides a two-times continuously-differentiable stiffness-angle relationship $\mathbf{K}(\mathbf{q}, \boldsymbol{\theta})$ in addition to motor-side positions $\boldsymbol{\theta}(t) \in \mathbb{C}^{2}$ generated by continuous motor torques.

\footnotetext{
${ }^{7} \mathbb{C}^{s}$ denotes the set of all $s$-times differentiable functions.

${ }^{8}$ Here, we assume that the motor positions may be generated by piecewisecontinuous motor torques that leads to continuously differentiable motor positions. This assumption asserts that the dynamics in the electrical domain associated with the motor-torque control is negligible on the time scale relevant in mechanical domain. This assumption is accepted here since it leads to the weakest continuity requirement that is physically justified in the present investigation.

${ }^{9}$ While it is possible, in principle, to design actuators that have nondifferentiable torque-angle characteristic (in which case the stiffness $\mathbf{K}=-\partial \boldsymbol{\tau} / \partial \mathbf{q}$
} may not be well defined) such designs are not considered here. 
On the other hand, torque and stiffness control may be instantaneous with respect to the time-scale relevant to the rigid body dynamics on noncompliant actuators. Accordingly, a similar condition to (20) does not restrict active impedance control on robots driven with (torque controllable) noncompliant actuators [36].

\section{ACTUATION CONSTRAINTS}

Conditions (17) and (19) may be seen as design constraints, while (20) provides fundamental physical constraints. There are, however, additional constraints that also restrict the output torque and stiffness of compliant actuators. In the following, we introduce these actuation constraints.

\section{A. Constraints on the Motor Trajectories}

Regardless of whether the system is compliantly actuated or not, the motor trajectories are subject to inequality constraints due to range, rate, and acceleration limits posed by their servocontrolled dynamics. The admissible set of trajectories subject to these constraints can be defined by

$$
\begin{aligned}
\Theta= & \left\{\boldsymbol{\theta} \in \mathbb{C}^{1}\left([0, T], \mathbb{R}^{m}\right): \boldsymbol{\theta}_{m} \preceq \boldsymbol{\theta}(t) \preceq \boldsymbol{\theta}_{M}\right. \\
& \left.|\dot{\boldsymbol{\theta}}(t)| \preceq \dot{\boldsymbol{\theta}}_{M},|\ddot{\boldsymbol{\theta}}(t)| \preceq \ddot{\boldsymbol{\theta}}_{M}\right\}
\end{aligned}
$$

where $\boldsymbol{\theta}_{m}$ and $\boldsymbol{\theta}_{M},\left(\boldsymbol{\theta}_{m} \prec \boldsymbol{\theta}_{M}\right)$ denote the lower and the upper bounds on the motor positions, while $\dot{\boldsymbol{\theta}}_{M}$ and $\ddot{\boldsymbol{\theta}}_{M}\left(\mathbf{0} \prec \dot{\boldsymbol{\theta}}_{M}, \mathbf{0} \prec\right.$ $\left.\ddot{\boldsymbol{\theta}}_{M}\right)$ are the achievable maximal velocities and accelerations, respectively. To be physically realizable, the motor trajectories should adhere to these limitations: $\boldsymbol{\theta}(t) \in \Theta$.

\section{B. Deformation Limits on the Compliant Elements}

In addition to the aforementioned constraints, series elastic actuators and variable stiffness devices may also posses nonlinear state inequality constraints, due to limits on the deformation of the compliant elements. The admissible set of the actuator positions that are associated with these constraints is formally defined by

$$
\begin{aligned}
\Phi= & \left\{\boldsymbol{\phi} \in \mathbb{C}^{1}\left(\mathbb{R}^{n} \times \mathbb{R}^{m}, \mathbb{R}^{m}\right):\right. \\
& \left.\boldsymbol{\phi}_{m}(\boldsymbol{\theta}) \preceq \boldsymbol{\phi}(\mathbf{q}, \boldsymbol{\theta}) \preceq \boldsymbol{\phi}_{M}(\boldsymbol{\theta})\right\}
\end{aligned}
$$

where $\phi$ denotes the quantities (e.g., length of the springs [see Fig. 2)] to be constrained during the motion, while $\phi_{m}$ and $\phi_{M}\left(\phi_{m} \prec \phi_{M}\right)$ define, the possibly state-dependent, lower and upper bounds.

It is noteworthy that violation of these constraints would not only lead to behavior that considerably differs from the planned one, but it would permanently damage the actuators. ${ }^{10}$ Accordingly, enforcing the aforementioned constraints, $\phi(\mathbf{q}, \boldsymbol{\theta}) \in \Phi$ may often be prioritized in real-world applications.

\footnotetext{
${ }^{10}$ In this case, the moving elements in the actuators would run into hard stops and/or the compliant element (spring) would be operated in the plastic region, and as such permanently deformed.
}

\section{OPTIMIZATION UNDER ACTUATION CONSTRAINTS}

Here, we present an optimal control formulation and an approach to trajectory optimization to plan control strategies best suited to the dynamics and the specificity of the task considered. Using this approach, we can systematically treat the complexity (i.e., nonlinearity, redundancy, and constraint) inherent to compliantly actuated robots. While finding control programs, i.e., desired motor trajectories (and the associated torque and passive impedance) under actuation constraints poses significant challenges, the present formulation is able to deal with this in a systematic way, without problem specific derivation or difficult computation.

In the following, we: 1) derive a minimalistic (physically consistent) model for compliantly actuated robots; 2) introduce the treatment of constraints; 3) propose an optimal control formulation; and finally 4) define the optimal motor program for hardware implementations.

\section{A. Minimalistic Model for Optimization}

In this section, we assume that conditions (C.2) and (C.3), stated in Section III-A, apply. Consistent with the former condition is Spong's assumption by which

A.3: the inertial coupling between the motor-side and the linkside dynamics is negligible, i.e., $\mathcal{T}_{12} \approx 0$

in (1), see [19].

Under (A.3) the following simplifications apply [see (1), and (5)-(7)]:

$$
\mathbf{S} \approx \mathbf{0}, \mathbf{C}=\mathbf{C}(\mathbf{q}, \dot{\mathbf{q}}), \mathbf{C}_{q} \approx \mathbf{0}, \mathbf{C}_{\theta} \approx \mathbf{0}
$$

As a consequence, the original equation (4) supplemented with the control law (12) reduces to

$$
\begin{aligned}
\mathbf{M}(\mathbf{q}) \ddot{\mathbf{q}}+\mathbf{C}(\mathbf{q}, \dot{\mathbf{q}}) \dot{\mathbf{q}}+\mathbf{D} \dot{\mathbf{q}}+\mathbf{G}(\mathbf{q}) & =\boldsymbol{\tau}(\mathbf{q}, \boldsymbol{\theta}) \\
\ddot{\boldsymbol{\theta}}+2 \boldsymbol{\kappa} \dot{\boldsymbol{\theta}}+\boldsymbol{\kappa}^{2} \boldsymbol{\theta} & =\boldsymbol{\kappa}^{2} \boldsymbol{\theta}_{d}
\end{aligned}
$$

where (25) resembles a critically damped ${ }^{11}$ closed-loop motor dynamics (15), where $\boldsymbol{\beta}=\boldsymbol{\kappa}$ (see Section III-A). We note that if the gear reduction is not particularly high [i.e., (A.3) does not apply] one should complement the link-side dynamics (24) with additional inertial terms indicated by (4). On the other hand, the validity of (25) is not conditioned on highly geared actuation as long as high-gain servocontrol is employed (see Section III). The purpose of this latter equation is to plan for realizable (smooth enough) motor trajectories, i.e., $\boldsymbol{\theta}$ that are consistent with the constraints. In this paper, we consider (24) and (25) to be a minimalistic representation of (4)-(10), which ensures the fundamental continuity conditions (20) for any, at least, piecewise-continuous desired trajectory $\boldsymbol{\theta}_{d}$.

\footnotetext{
${ }^{11}$ It may be important to note here that assuming critical-damped closed-loop motor dynamics is not restrictive. This is partly because critical damping is often preferred in trajectory tracking schemes (especially for systems driven be compliant actuators), but also because the subsequent results, that depend on this assumption, can be generalized to overdamped systems (i.e., $\boldsymbol{\beta} \succ \boldsymbol{\kappa}$ ).
} 


\section{B. Treatment of Constraints}

While incorporating the constraints (21) and (22), into the optimization is vital, numerical treatment of them is nontrivial. Indeed, solving an optimal control problem with state inequality constraints is often computationally demanding and complicates the numerical treatment (see Section IX). Moreover, excessive application of constraints makes the associated formulation more susceptible to local-minima issues. Finding an optimal solution to such a formulation is known to be difficult. Therefore, the reduction of the number and complexity of the constraints is often preferred in practice. Here, we address this issue by replacing nonlinear state inequality constraints with canonical control constraints that are easier to treat numerically. To achieve this, we present a differential-algebraic transformation that enables us to explicitly embed number of inequality constraints as hard constraints (i.e., constraints that cannot be violated) into the dynamics.

1) Transforming the Constraints: Let us now consider a set of user defined nonlinear inequalities

$$
\begin{aligned}
\Psi= & \left\{\boldsymbol{\psi} \in \mathbb{C}^{1}\left(\mathbb{R}^{n} \times \mathbb{R}^{m}, \mathbb{R}^{m}\right):\right. \\
& \left.\boldsymbol{\psi}_{m}(\boldsymbol{\theta}) \preceq \boldsymbol{\psi}(\mathbf{q}, \boldsymbol{\theta}) \preceq \boldsymbol{\psi}_{M}(\boldsymbol{\theta})\right\}
\end{aligned}
$$

where $\Psi$ denotes the admissible set of quantities $\psi \in \mathbb{R}^{m}$ (e.g., including motor positions $\boldsymbol{\theta}$ and/or spring lengths $\phi$ ) to be constrained during the motion, while $\boldsymbol{\psi}_{m} \prec \boldsymbol{\psi}_{M}$ are the associated (possibly state-dependent) lower and upper bounds. We assume that these bounds are also continuously differentiable functions of their arguments.

In a special case when $\psi=\boldsymbol{\theta},(26)$ reduces to the range constraints on the motor positions, while in the other extreme case when $\boldsymbol{\psi}=\boldsymbol{\phi}(\mathbf{q}, \boldsymbol{\theta})$, (26) would reduce to (22). To embed these constraints into the dynamics, we may proceed in the following way:

1) define a canonical input set ${ }^{12}$

$$
Z_{d}=\left\{\mathbf{z}_{d} \in P C\left([0, T], \mathbb{R}^{m}\right): \mathbf{0} \preceq \mathbf{z}_{d} \preceq \mathbf{1}\right\}
$$

2) then introduce a set of canonical state variables

$$
z_{i}=\frac{\psi_{i}-\psi_{i m}}{\psi_{i M}-\psi_{i m}}
$$

3) finally generate $\mathbf{z}=\left[z_{1}, z_{2}, \ldots, z_{m}\right]^{T}$ through a secondorder critically damped differential equation

$$
\ddot{\mathbf{z}}+2 \boldsymbol{\alpha} \dot{\mathbf{z}}+\boldsymbol{\alpha}^{2} \mathbf{z}=\boldsymbol{\alpha}^{2} \mathbf{z}_{d}
$$

where $\forall i \in\{1,2, \ldots, m\}, \alpha_{i}>0, \alpha^{2}=\operatorname{diag}\left\{\alpha_{i}^{2}\right\}$ and $z_{i}(0) \in[0,1]$ is defined by (28) based on the initial state of the system.

This differential-algebraic transformation, (28) and (29), provides constraint consistent state output for any admissible control input

$$
\forall \mathbf{z}_{d} \in Z_{d} \Rightarrow \psi \in \Psi
$$

It may be of interest to recognize that this transformation is not problem specific, but its application requires one parameter $\boldsymbol{\alpha}$ to

\footnotetext{
${ }^{12} P C\left([0, T], \mathbb{R}^{m}\right)$ denotes a set of piecewise continuous functions mapping $[0, T]$ to $\mathbb{R}^{m}$.
}

be set for every embedded constraint; this is detailed in Sections VI-C1 and VI-C2.

2) Computing the Motor Trajectories: One of the main questions regarding the proposed transformation is how to include the new state variables $\mathbf{z}$ into the dynamics (24) and (25). To this end, we consider (28) to be a coordinate transformation that relates $\mathbf{z}$ with $\boldsymbol{\theta}$ and $\mathbf{q}$ through the following relation:

$$
\Psi(\mathbf{q}, \boldsymbol{\theta}, \mathbf{z})=\mathbf{0}
$$

where $\boldsymbol{\Psi}(\mathbf{q}, \boldsymbol{\theta}, \mathbf{z}):=\boldsymbol{\psi}(\mathbf{q}, \boldsymbol{\theta})-\operatorname{diag}\left(\boldsymbol{\psi}_{M}(\boldsymbol{\theta})-\boldsymbol{\psi}_{m}(\boldsymbol{\theta})\right) \mathbf{z}-$ $\boldsymbol{\psi}_{m}(\boldsymbol{\theta})$. Using (31), we may find the motor trajectories $\boldsymbol{\theta}$ as functions of the canonical states $\mathbf{z}$ (and $\mathbf{q}$ ). Indeed, according to the implicit function theorem [49], one can (at least locally) define the following inverse mapping of (31)

$$
\boldsymbol{\theta}=\boldsymbol{\Psi}_{\theta}^{-1}(\mathbf{q}, \mathbf{z})
$$

for every $(\mathbf{q}, \mathbf{z})$ for which $\partial \boldsymbol{\Psi} / \partial \boldsymbol{\theta} \in \mathbb{R}^{m \times m}$ is a full-rank matrix. i.e.,

$$
\operatorname{det}(\partial \boldsymbol{\Psi} / \partial \boldsymbol{\theta}) \neq 0
$$

In addition, as long as $\boldsymbol{\psi}, \boldsymbol{\psi}_{m}$, and $\boldsymbol{\psi}_{M}$ are continuously differentiable with respect to their arguments (as assumed), the same is true for the inverse mapping $\Psi_{\theta}^{-1}(\mathbf{q}, \mathbf{z})$, and the motor trajectories $\boldsymbol{\theta}$ that are computed using (32).

It is important to note that fulfillment of (33) depends on the constraints. In particular, if (26) represents the constraints on the motor trajectories, the inverse mapping $\Psi_{\theta}^{-1}$ exist unconditionally (this is the case for the model of the two-link arm driven by mechanically adjustable compliance and controllable equilibrium position actuators (MACCEPAs) used in Section VII-B [see also Section VI-C1)]. It is also important to point out that the proposed scheme favors constraints that admit an analytical representation of $\Psi_{\theta}^{-1}$ and as such explicit computation of the motor trajectories [as in the model of the DLR Hand-Arm System used in Section VII-C (see also Section VI-C2)]. When this is not possible, one may employ Newton's method [50] to solve (31) for $\boldsymbol{\theta}$ directly. As long as the inverse mapping is well defined, i.e., (33) holds, the associated iterative computation can be well initialized and reliably solved with little computational effort. ${ }^{13}$

Finally, we note that the number of state inequality constraints that can be treated in this way is defined by the number of control inputs (motor positions) $m$. In the case when only the motor position are constrained, this allows full embedding of the constraints (discussed in Section VI-C1). In the case when deformation limits are also present, the proposed embedding can be used to reduce the number of constraints to be treated by a complementary approach. We note that in such a case, the proposed embedding may be employed on the complex deformation limits (22) that are vital to be enforced, while leaving the simpler motor position constraints to be treated separately (as exemplified in Section VII-C).

\footnotetext{
${ }^{13}$ This is because one can effectively initialize Newton's method for the computation of the motor positions $\boldsymbol{\theta}\left(t_{n+1}\right)$ using the nearby solution for the previous time step $\boldsymbol{\theta}\left(t_{n}\right)$, since $\boldsymbol{\theta}=\boldsymbol{\theta}(t)$ defined by (32) is a continuously differentiable function.
} 


\section{State-Space Representation}

Let us now formally introduce the state vector and the control inputs as

$$
\mathbf{x}=\left[\begin{array}{c}
\mathbf{x}_{1} \\
\mathbf{x}_{2} \\
\mathbf{x}_{3} \\
\mathbf{x}_{4}
\end{array}\right]=\left[\begin{array}{c}
\mathbf{q} \\
\dot{\mathbf{q}} \\
\mathbf{z} \\
\dot{\mathbf{z}}
\end{array}\right], \mathbf{u}=\mathbf{z}_{d}
$$

where $\mathbf{x} \in \mathbb{R}^{2(n+m)}$ and $\mathbf{u}$ belongs to a canonical boxconstrained set

$$
U=\left\{\mathbf{u} \in P C\left([0, T], \mathbb{R}^{m}\right): \mathbf{0} \preceq \mathbf{u} \preceq \mathbf{1}\right\} .
$$

Using this notion, the state-space representation of the original constrained system (24)-(26) becomes

$$
\mathbf{f}=\left[\begin{array}{c}
\dot{\mathbf{x}}=\mathbf{f}(\mathbf{x}, \mathbf{u}) \\
\mathbf{x}_{2} \\
-\mathbf{M}^{-1}\left(\mathbf{x}_{1}\right)\left(\mathbf{C}\left(\mathbf{x}_{1}, \mathbf{x}_{2}\right) \mathbf{x}_{2}+\mathbf{D} \mathbf{x}_{2}+\mathbf{G}\left(\mathbf{x}_{1}\right)-\mathbf{T}\left(\mathbf{x}_{1}, \mathbf{x}_{3}\right)\right) \\
\mathbf{x}_{3} \\
-2 \boldsymbol{\alpha} \mathbf{x}_{4}-\boldsymbol{\alpha}^{2} \mathbf{x}_{3}+\boldsymbol{\alpha}^{2} \mathbf{u}
\end{array}\right]
$$

where $\mathbf{T}=\mathbf{T}\left(\mathbf{x}_{1}, \mathbf{x}_{3}\right)$ denotes the actuator torques that are expressed through the new state variables

$$
\mathbf{T}=\mathbf{T}\left(\mathbf{x}_{1}, \mathbf{x}_{3}\right)=\mathbf{T}(\mathbf{q}, \mathbf{z})=\boldsymbol{\tau}\left(\mathbf{q}, \Psi_{\theta}^{-1}(\mathbf{q}, \mathbf{z})\right) .
$$

Taking these together, the proposed formulation is fully specified with (36) and (37), where the control inputs are restricted with the simplest set of inequality constraints (i.e., canonical box-constraints) e.g., (35), but it does not involve any state inequality constraints e.g., (26).

In order to employ this formulation, however, one must define one parameter for every embedded constraint $\boldsymbol{\alpha}=\operatorname{diag}\left\{\alpha_{i}\right\}$. If these parameters are positive, $\forall \alpha_{i}>0$, then the range constraints (26) cannot be violated. ${ }^{14}$ In the following, we give a physical interpretation of $\boldsymbol{\alpha}$, and discuss how to select this parameter in two important practical cases of interest.

1) Embedding Bandwidth Limitations on the Motor Dynamics: Let us now assume that, for a given actuator, there are no complex state inequality constraints (e.g., there are no limitations on the elastic deformation of the springs), or even if such limits exist, they are not expected to be violated during the motion. ${ }^{15}$ In that case, any admissible motor trajectory must obey the range, rate, and acceleration limits given by (21)

$$
\boldsymbol{\theta} \in \Theta .
$$

Using the formulation in the previous section, we can enforce the associated range limits $\boldsymbol{\theta}_{m} \preceq \boldsymbol{\theta} \preceq \boldsymbol{\theta}_{M}$, by setting

a) $\boldsymbol{\psi}=\boldsymbol{\theta}, \boldsymbol{\psi}_{m}=\boldsymbol{\theta}_{m}, \boldsymbol{\psi}_{M}=\boldsymbol{\theta}_{M}$.

In this case, the inverse mapping (32) can be explicitly defined

$$
\boldsymbol{\theta}=\boldsymbol{\Psi}_{\theta}^{-1}(\mathbf{z})=\operatorname{diag}\left(\boldsymbol{\theta}_{M}-\boldsymbol{\theta}_{m}\right) \mathbf{z}+\boldsymbol{\theta}_{m} .
$$

\footnotetext{
${ }^{14}$ This follows directly from $\mathbf{u} \in U \Rightarrow \mathbf{0} \preceq \mathbf{x}_{3}=\mathbf{z} \preceq \mathbf{1}$ and (28).

${ }^{15}$ This assumption may be ensured through design as is done for our two-link variable stiffness robot introduced in Section VII-B.
}

Direct implication of this relation is that, for a critically damped motor dynamics, $\boldsymbol{\alpha}$ coincides with the position gains $\boldsymbol{\kappa}$ in (25). Accordingly, we can ensure that not only the range constraints but also the bandwidth limits that are associated with the rate and acceleration constraints $|\dot{\boldsymbol{\theta}}| \preceq \dot{\boldsymbol{\theta}}_{M}$ and $|\ddot{\boldsymbol{\theta}}| \preceq \ddot{\boldsymbol{\theta}}_{M}$ are satisfied by choosing

b) $\alpha_{i} \in\left(0, \alpha_{i M}\right], \forall i \in\{1,2, \ldots, m\}$ where

$$
\alpha_{i M}=e \times \min \left\{\frac{\dot{\theta}_{i M}}{\theta_{i M}-\theta_{i m}}, \sqrt{\frac{\ddot{\theta}_{i M}}{\theta_{i M}-\theta_{i m}}}\right\} .
$$

Note that embedding the velocity and acceleration limits is indeed possible in this way since $\boldsymbol{\alpha}_{M}$ represents the maximal position gains that guarantee (21), under piecewise-continuous inputs $\mathbf{u} \in U$. Regarding the choice of $\alpha_{i}$, we note that any $\alpha_{i} \in\left(0, \alpha_{i M}\right]$ would ensure (21), however, $\alpha_{i}=\alpha_{i M}$ would lead to the best exploitation of the motor-side dynamics and as such it may be preferred in practice. ${ }^{16}$ It is noteworthy that under conditions a) and b) introduced in this section, the formulation (34)-(37) reduces to the one proposed in [18]. For further application of this formulation, see [41].

2) Embedding Deformation Limits on the Elastic Elements: Let us now consider another case when the length of the springs in the actuators have to be constrained during the motion. In this case, we may require

$$
\phi(\mathbf{q}, \boldsymbol{\theta}) \in \Phi .
$$

Using the proposed formulation (35)-(37), the aforementioned restriction can be ensured by setting $\psi=\phi(\mathbf{q}, \boldsymbol{\theta})$. In that case, the canonical states $\mathbf{z}$ represent the normalized spring lengths

$$
\boldsymbol{\phi}(\mathbf{q}, \boldsymbol{\theta})=\operatorname{diag}\left(\boldsymbol{\phi}_{M}(\boldsymbol{\theta})-\boldsymbol{\phi}_{m}(\boldsymbol{\theta})\right) \mathbf{z}+\boldsymbol{\phi}_{m}(\boldsymbol{\theta}) .
$$

The inverse mapping $\boldsymbol{\theta}=\boldsymbol{\Psi}_{\theta}^{-1}(\mathbf{q}, \mathbf{z})$, that solves this relation for $\boldsymbol{\theta}$, is in general link-side position dependent and may be hard to define explicitly. A notable exception to this is when the spring length is a linear function of a motor-side position. For example, $\phi_{i}=\theta_{i}-q_{i}$ as in elastic joint robots or in the model of the DLR hand-arm system (see Section VII-C). In such cases, the motor positions can be explicitly defined: $\theta_{i}=$ $\psi_{\theta}^{-1}\left(q_{i}, z_{i}\right)=q_{i}+\left(\phi_{i M}-\phi_{i m}\right) z_{i}+\phi_{i m}$ (as long as $\phi_{i M}$ and $\phi_{i m}$ do not depend on $\left.\theta_{i}\right)$.

Regardless of whether one can explicitly compute the motor trajectories, the dynamics that are associated with $\mathbf{z}$ [third and fourth lines in (36)] defines the dynamics of the normalized spring motion. The physical implication of this is that, here $\boldsymbol{\alpha}$ limits the bandwidth of the entire system (i.e., link-side and motor-side dynamics), and as such it cannot be used to embed the bandwidth limitations on the actuators alone. In this light, the velocity and acceleration limits $|\dot{\boldsymbol{\theta}}| \preceq \dot{\boldsymbol{\theta}}_{M}$ and $|\ddot{\boldsymbol{\theta}}| \preceq \ddot{\boldsymbol{\theta}}_{M}$

\footnotetext{
${ }^{16}$ It is also noteworthy that $\alpha_{i M}$ computed from (40) often turns out to be conservative. There are two main reasons to this. One is because $\alpha_{i M}$ is calculated assuming a maximal step response command $\theta_{i M}-\theta_{i m}$, although this may not be the usual command in practice, while the another is because real servo-systems are often deliberately pushed to saturation to improve their tracking performance compared with an unsaturated ideal linear servo model [51]. Due to these reason one may chose $\alpha_{i}>\alpha_{i M}$ as long as this does not jeopardize the tracking accuracy.
} 
cannot be embedded into the formulation, in a way described in the previous section, and as such remain to be treated by a complementary approach. In Section VII-C, we illustrate this through simulations and a practical application.

\section{Optimal Control Problem}

In order to define optimal control strategies for compliantly actuated robots, we consider the following control problem. For a given finite time interval $t \in[0, T]$, and for a given initial state of the system $\mathbf{x}(0)=\mathbf{x}_{0}$, find the control inputs $\mathbf{u}$ that minimize the optimization criterion defined by

$$
J=h(T, \mathbf{x}(T))+\int_{0}^{T} c(t, \mathbf{x}(t), \mathbf{u}(t)) d t
$$

where $J \in \mathbb{R}$ is the cost functional, $h(T, \mathbf{x}(T)) \in \mathbb{R}$ is the terminal cost, while $c(t, \mathbf{x}, \mathbf{u}) \in \mathbb{R}$ is the running cost used to encode the control objectives into the formulation [52]. This minimization problem is restricted with the plant dynamics

$$
\dot{\mathbf{x}}=\mathbf{f}(\mathbf{x}, \mathbf{u})
$$

and the control constraints (i.e., canonical box-constraints)

$$
\mathbf{u} \in U
$$

defined by (35)-(37).

In this paper, we employ the iLQR framework [53] in order to find the optimal solutions $\mathbf{x}^{*}=\mathbf{x}^{*}(t)$ and $\mathbf{u}^{*}=\mathbf{u}^{*}(t)$ for the constrained optimal control problem defined by (43)(45). A discussion on alternative methods to solve this problem is provided in the Appendix. In the following, we show how to compute the desired motor trajectories required in practical implementations, once the optimal state trajectories are found.

\section{E. Computing the Desired Motor Trajectories}

If the constraints on the actuators (21) are incorporated into the formulation, the motor trajectories computed by (32), i.e., $\boldsymbol{\theta}^{*}=\boldsymbol{\Psi}_{\theta}^{-1}\left(\mathbf{q}^{*}(t), \mathbf{z}^{*}(t)\right)=\boldsymbol{\Psi}_{\theta}^{-1}\left(\mathbf{x}_{1}^{*}(t), \mathbf{x}_{3}^{*}(t)\right)$, will be within the bandwidth of the actuators. This means that by using servocontrol on the motor side [see (15) or (25)], with optimal desired trajectories computed by

$$
\boldsymbol{\theta}_{d}=\boldsymbol{\Psi}_{\theta}^{-1}\left(\mathbf{q}^{*}(t), \mathbf{z}^{*}(t)\right)=\boldsymbol{\Psi}_{\theta}^{-1}\left(\mathbf{x}_{1}^{*}(t), \mathbf{x}_{3}^{*}(t)\right)
$$

will ensure that the real motor positions $\boldsymbol{\theta}$ closely follow the corresponding optimal motion $\boldsymbol{\theta}^{*}$, i.e.,

$$
\boldsymbol{\theta} \approx \boldsymbol{\theta}_{d}=\boldsymbol{\theta}^{*} .
$$

It is noteworthy that this argument does not rest on the linearity of the closed-loop controlled motor dynamics. As such it remains valid, even if the servosystem is deliberately pushed into saturation to provide better tracking performance [51].

\section{APPLICATION}

In this section, we provide numerical simulations and hardware experiments to demonstrate the viability of the proposed optimal control formulation. These simulations and experiments involve two redundantly actuated variable stiffness robots, specifically:
1) the two-link robot [18], shown in Fig. 3(a), driven by MACCEPAs [54] [see Fig. 3(b) and (c)];

2) the integrated DLR hand-arm system (HASy) [1], shown in Fig. 7, equipped with variable stiffness floating spring joints (FSJ) [7] [see Fig. 5(a) and (b)].

The purpose of the presented numerical study is to illustrate the effect of the actuation constraints on robotic systems driven by compliant actuators. The purpose of the hardware experiments is to demonstrate the viability of the constraint embedding method and the proposed optimal impedance control planning under real-world conditions.

\section{A. Control Task}

The following investigation involves a ball throwing task. This task is associated with fast movements, where exploitation of the full capacity of the actuators, under given constraints, is important to improve task performance (i.e., distance thrown). Within the framework of optimal control, ball throwing is represented with the following objective functional (see [18]):

$$
J_{t}=-d+\frac{1}{2} \int_{0}^{T}\left(w\|\mathbf{F}\|^{2}+\epsilon\|\mathbf{u}\|^{2}\right) d t
$$

where $T$ is the time permitted to task execution, $d=$ $d(\mathbf{q}(T), \dot{\mathbf{q}}(T))$ is the distance thrown, $\mathbf{F}=\mathbf{F}(\mathbf{q}, \boldsymbol{\theta})$ is the spring force, $\mathbf{u}$ is the control input while $\|*\|$ denotes the Euclidean norm.

Here, we consider a predominantly distance maximization task characterized with small effort penalization (i.e., $w \rightarrow 0$ ), and a small regularization term (i.e., $0<\epsilon \ll 1$ ) that makes the objective explicitly control-dependent and the control problem nonsingular. It is important to note that the optimal strategy should adhere to the actuation constraints (21) and (22), since it will only be physically realizable if these constraints are satisfied.

\section{B. Two-Link Variable Stiffness Robot}

The variable stiffness robot, shown in Fig. 3, has two kinematic DOFs, and is equipped with two VSAs driven with four motors. The configuration of this system is defined with the corresponding joint angles $\mathbf{q}=\left[q_{1}, q_{2}\right]^{T}$ and motor angles $\boldsymbol{\theta}=\left[\theta_{1}, \theta_{2}, \theta_{3}, \theta_{4}\right]^{T}$. One of the motors in each VSAs $\left(\theta_{1}\right.$ and $\left.\theta_{2}\right)$ changes the joint equilibrium, while the another $\left(\theta_{3}\right.$ and $\theta_{4}$ ) modulates the passive joint stiffness. In Fig. 3(c)-(e), we show the actuation mechanism, and the torque-angle and torquestiffness characteristics of these actuators.

1) Simulation and Experimental Results: In Fig. 4(a) and (b), we show the effect of the actuators range and rate (bandwidth) limitation on the optimal control strategy. It can be recognized that using larger stiffness range $\theta_{3,4} \in[0, \pi / 2] \mathrm{rad}$ and fast (high-bandwidth) actuators for stiffness modulation $\alpha_{3,4}=$ $5[\sqrt{10}, \sqrt{10}] \mathrm{s}^{-1}$, instead of small ranges $\theta_{3,4} \in[0, \pi / 4] \mathrm{rad}$ and slow (low-bandwidth) actuators $\alpha_{3,4}=5[1,1] \mathrm{s}^{-1}$, not only affects the systems performance, but it can also qualitatively modify the stiffness control strategy. Specifically, when the actuators are slow, active modulation of the torque-stiffness characteristics of the joints appear not to be beneficial [see gray lines in 
(a)

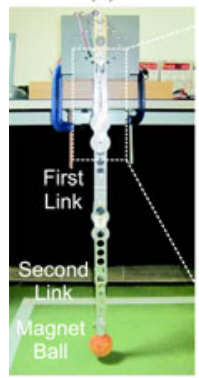

(b)

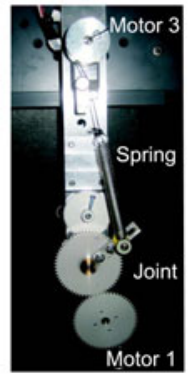

(c)

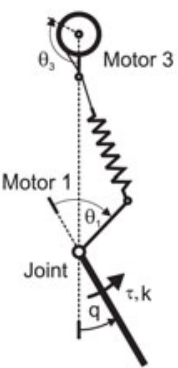

(d)

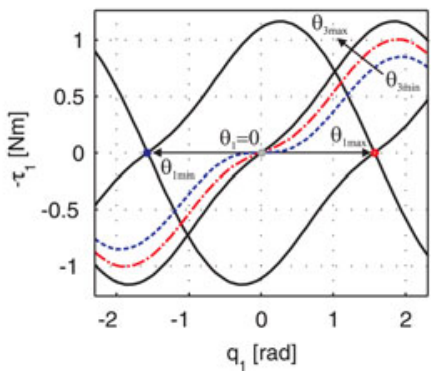

(e)

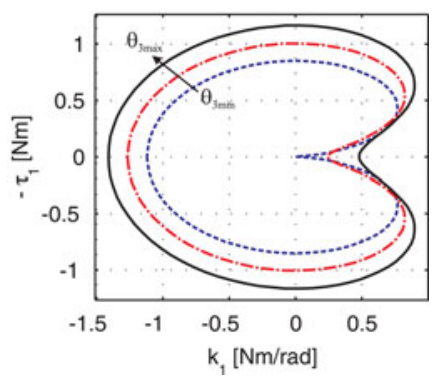

Fig. 3. (a) Two-link variable-stiffness robot. (b) and (c) Variable-stiffness actuator. The physical parameters of this system are given in [18]. (d) Torque-angle curves. (e) Torque-stiffness curves. Each of these curves define the passive torque-stiffness characteristic of the joint corresponding to constant stiffness commands $\theta_{3}=c$ (see Sections IV-A and IV-B). By changing the equilibrium point $\theta_{1}=\theta_{1}(t)$ but keeping the pretension commands fixed $\theta_{3}=c$, one can move along these passive isocurves in the torque-stiffness plane. However, by changing the pretensioning continuously in time $\theta_{3}=\theta_{3}(t)$, one can also modify these curves during the movement. This ability to actively modify the passive torque-stiffness characteristics of the joint allows simultaneous torque and stiffness modulation. Note that by keeping $\theta_{3}=c$, this VSA becomes a series elastic actuator that has: 1) nonlinear torque-angle characteristic; and 2) fixed torque-stiffness characteristic regardless of $\theta_{1}=\theta_{1}(t)$. This means that under $\theta_{3}=c$, this actuator cannot provide independent torque and stiffness modulation. This can be easily verified using (19). On the other hand, by fixing $\theta_{1}=c$ but changing $\theta_{3}=\theta_{3}(t)$, the actuator can generate different torque-stiffness characteristics, but it may not be able to generate any output torque, e.g., if $\theta_{1}=q_{1}$. This may be verified using (17).
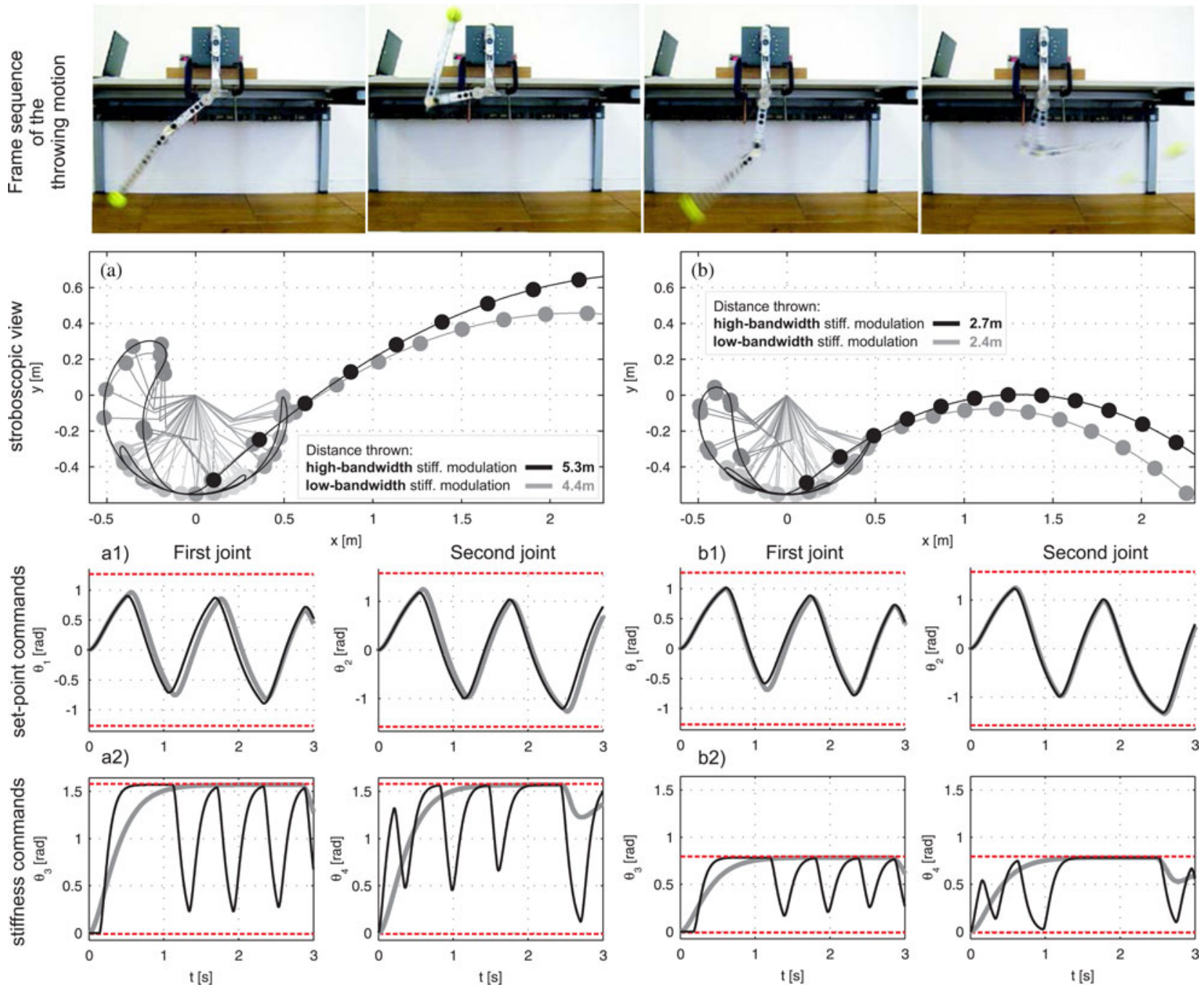

Fig. 4. Ball throwing using: (a, a1, a2) actuators having large stiffness range $\theta_{3,4} \in[0, \pi / 2]$ rad and high bandwidth $\boldsymbol{\alpha}=5 \operatorname{diag}([1,1, \sqrt{10}, \sqrt{10}]) \mathrm{s}^{-1}$ (black lines) or low bandwidth $\boldsymbol{\alpha}=5 \operatorname{diag}([1,1,1,10]) \mathrm{s}^{-1}$ (gray lines); (b, b1, b2) actuators having small stiffness range $\theta_{3,4} \in[0, \pi / 4] \mathrm{rad}$ and high bandwidth $\boldsymbol{\alpha}=5 \operatorname{diag}([1,1, \sqrt{10}, \sqrt{10}]) \mathrm{s}^{-1}$ (black lines) or low bandwidth $\boldsymbol{\alpha}=5 \operatorname{diag}([1,1,1,1]) \mathrm{s}^{-1}$ (gray lines). Limits on the motor positions (depicted with dashed red lines) are set to: $\theta_{1,2} \in[-2 \pi / 5,2 \pi / 5]$ rad. Practical implementation of the optimally planned motor program, corresponding to high-bandwidth and large stiffness range actuators [see Fig. 4(a)_black line], is demonstrated in the supplementary material. For further experiments on this system (and a similar one-link device) [see [18] and [16] respectively]. 
(a)

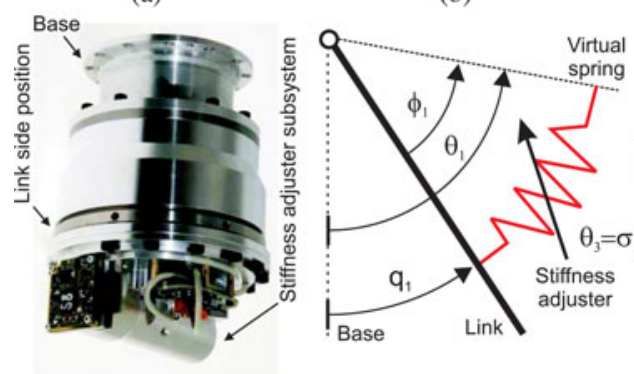

(c)

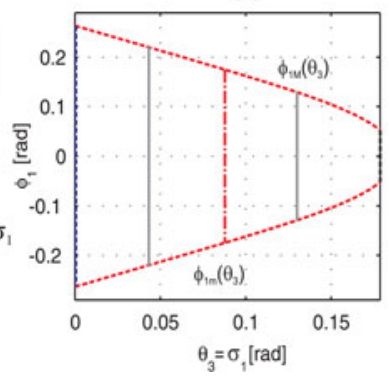

(d)

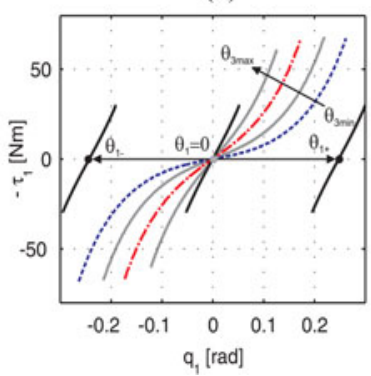

(e)

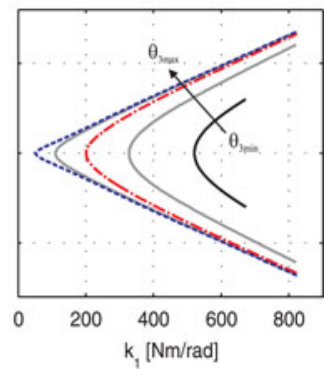

Fig. 5. (a) DLR floating spring joint [7]. (b) Schematic representation: $q_{1}$ is the joint angle, $\theta_{1}$ is the motor angle that is associated with the equilibrium position of the virtual rotational joint spring, $\phi_{1}=\theta_{1}-q_{1}$ is the length of the virtual spring, while $\theta_{3}$ is the motor angle that is associated with the stiffness of this spring. (c) Deformation limit of the spring give rise to nonlinear (stiffness command dependent) inequality constraints: $\phi_{1 m}\left(\theta_{3}\right) \leq \phi_{1} \leq \phi_{1 M}\left(\theta_{3}\right)$. (d) Torque-angle characteristics. (e) Torque-stiffness characteristics. Note that, by setting $\theta_{3}=c$, the DLR-FSJ behaves as a nonlinear spring with controllable joint equilibrium angle $\theta_{1}$, while by setting $\theta_{1}=c$, it becomes a joint that could have different stiffness properties depending on $\theta_{3}$. It is of practical interest to recognize that a single torque-stiffness curves indicates that the actuator is not capable of independent torque/stiffness modulation (i.e., SEA), while multiple curves (that enclose a region in the torque-stiffness plane [see Fig. 3(e) and Fig. 5(e)] indicate that independent torque/stiffness modulation is viable (i.e., VSA). Accordingly, visual inspection of the torque-stiffness characteristics may be used as an alternative to the analytical condition (19).

Fig. 4(a2) and (b2)], as opposed to the case when the same task is realized with actuators capable of fast stiffness modulation [see black lines in Fig. 4(a2) and (b2)]. Moreover, when the stiffness range is smaller, the difference between variable and constant stiffness control becomes less apparent. These results illustrate the crucial effects of various actuation constraint on optimal (impedance/stiffness) control strategies and support our claim by which these constraints are vital to be considered during control planning.

2) Discussion: It is clear that having a limited stiffness range, the benefit that is provided by variable stiffness optimization compared with constant stiffness control, may be negligible (e.g., see (26) in [39]). It is however less obvious that the limitation on the speed of the stiffness modulation can significantly impact the effect of variable stiffness control in dynamic tasks. At the extreme, optimal stiffness control may predict that active stiffness modulation is not beneficial if the speed of this modulation is slow compared with the dynamics of the movement [18]. This is to say that the optimal strategy is not only task specific (i.e., that relates to the objective functional), or specific to the rigid body dynamics (that relates to the first equation in (4) [or (24)]), but it can significantly depend on the actuator dynamics (second equation in (4) [or (25)]) and the actuation constraints i.e., (21) and (22). Due to this reason, there is an obvious danger that the actuator dynamics (when slow) filters out a stiffness modulation that may be intuitively expected for a given task, but also that some actuator-specific stiffness control feature are mistakenly attributed as task induced.

There are important implications of this to: 1) new compliant actuator designs; and 2) interpretations concerning human impedance control. Regarding actuator designs, it becomes apparent that not only the range but also the dynamics (i.e., bandwidth) related to stiffness modulation needs to be part of the design specification. This is especially important for dynamic tasks, where stiffness modulation must be fast enough to become relevant on the time scale set by the task and the link-side dynamics. If this condition is not met, the benefit that may come from simultaneous torque and impedance modulation will be lost. Accordingly, just because a variable impedance actuator is capable of torque and impedance modulation, it does not mean that this feature will be beneficial. This could be due to the task itself, characteristic of the link-side dynamics, but also due to the constraints imposed by the actuators. We note that while high-bandwidth actuation may not be vital in static tasks [55], it becomes important and highly relevant in dynamic tasks. This should be taken into account when interpreting human stiffness (impedance) control strategies that is subject to realistic biological (e.g. muscle-tendon) bandwidth-limitations [56]. Regarding this last point, we note that care should also be taken when transferring experimental observations from humans to robots or vice versa [57]. This is because impedance strategies by humans are subject to inherent bandwidth limitations of the muscle-tendon system and as such, may not contain important task-specific features that could be utilized by robots equipped with fast actuators. This is to say that biological control strategies could be very different from those optimal for robots (even if both are obtained through optimization of the same objective functional).

\section{DLR Hand-Arm System}

The DLR-HASy is a state-of-the-art variable stiffness robot that resembles the complexity of a human upper limb (see Fig. 7). For the purpose of the present investigation, the simulation/experimental results conducted with this device, are reported in the sagittal plane, namely the shoulder and elbow rotation joint (i.e., $\mathbf{q}=\left[q_{1}, q_{2}\right]^{T}$ ). Both of these joints incorporate variable stiffness actuators [see Fig. 5(a)]. These VSAs involve a main motor $\left(\theta_{1}\right.$ and $\left.\theta_{2}\right)$ that modulate the set point of the virtual spring and a small motor $\left(\theta_{3}\right.$ and $\left.\theta_{4}\right)$ that adjusts the joint stiffness [see Fig. 5(b)]. In Fig. 5(c) and (d), we show the deformation limits and the torque-angle characteristics of these actuators, while in Fig. 5(e), we show the torque-stiffness characteristics of the joints.

The range/rate constraints that are associated with the limitations on the position and velocities of the stiffness adjuster motors $\boldsymbol{\sigma}=\left[\theta_{3}, \theta_{4}\right]^{T}$ and the deformation limits on the floating 
(a)
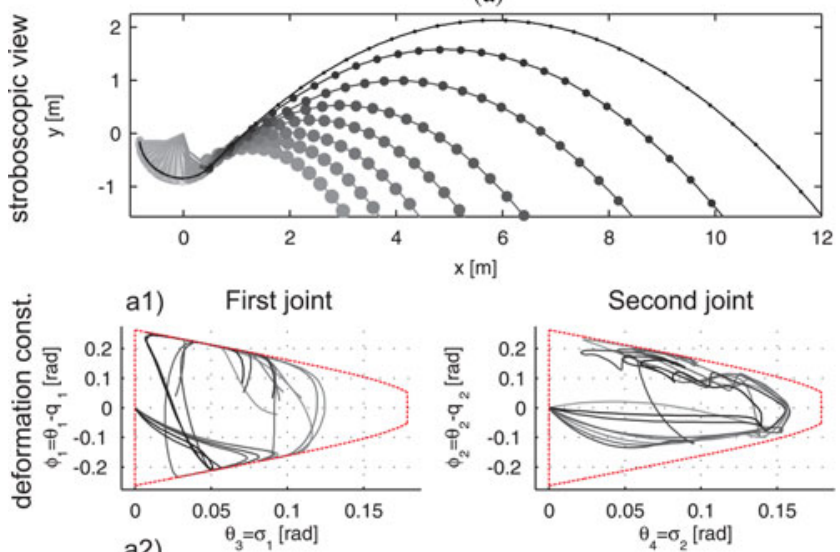

a2)
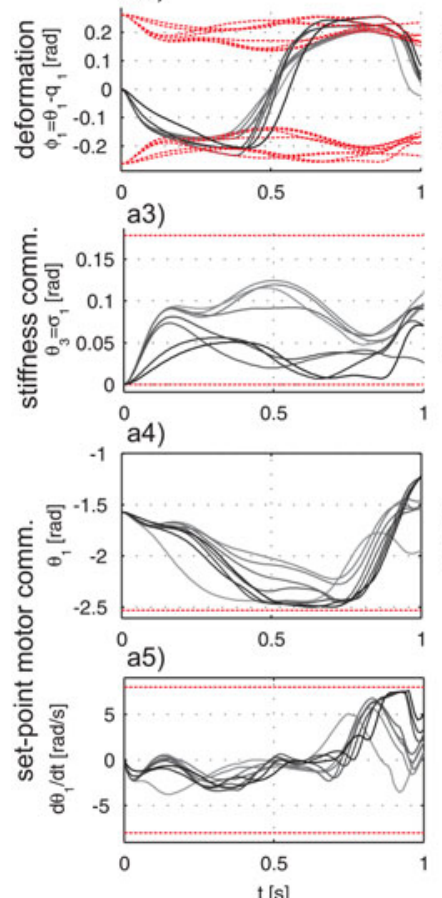

Second joint
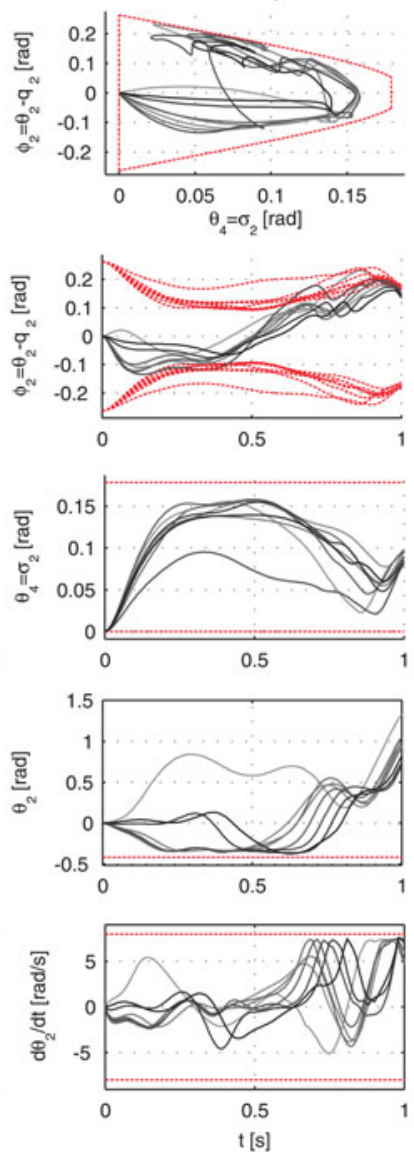

(b)

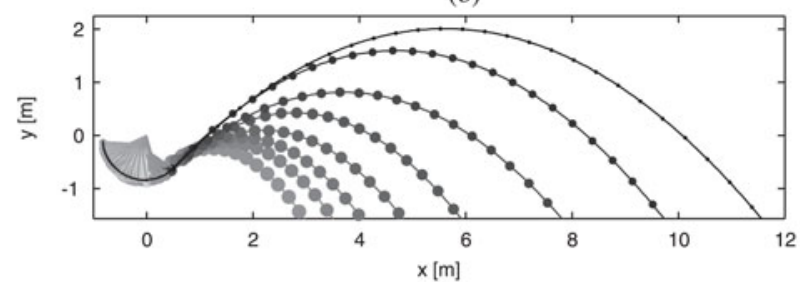

b1) First joint
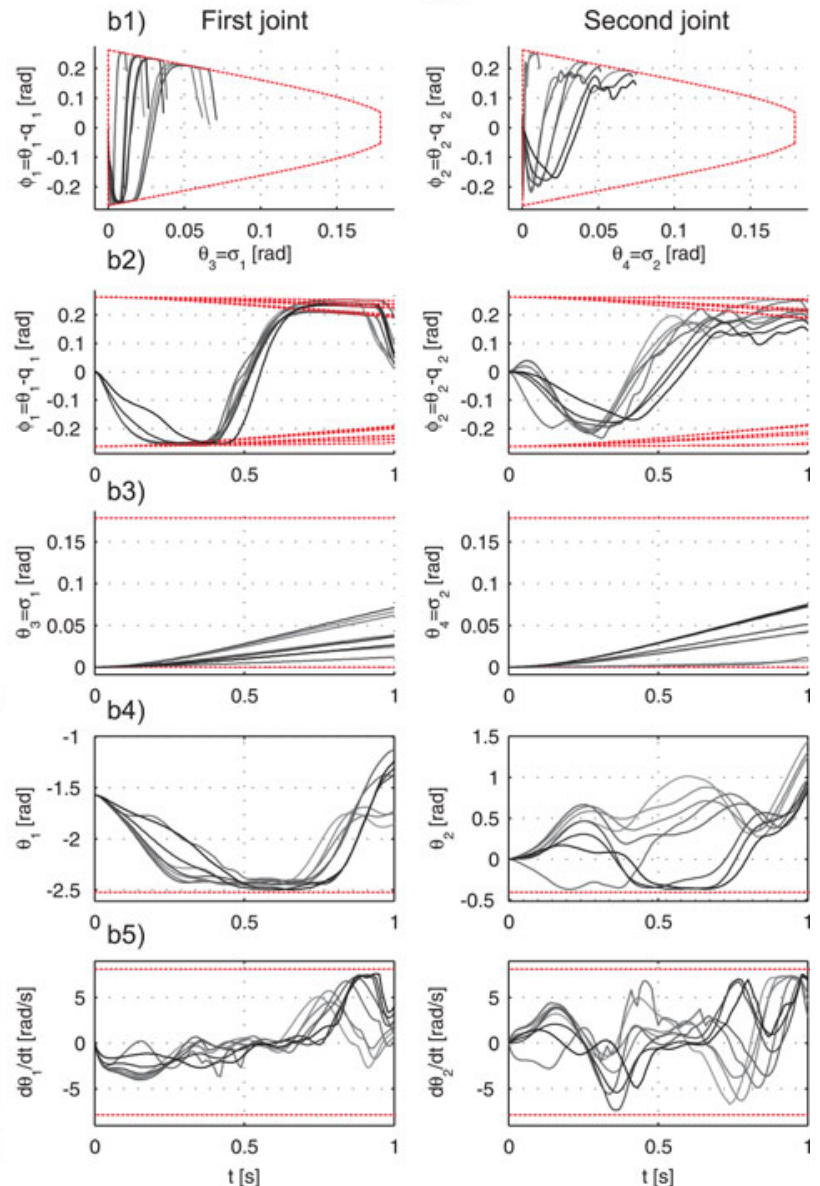

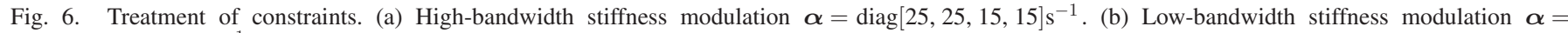
$\operatorname{diag}[25,25,1.5,1.5] \mathrm{s}^{-1}$. The simulations are performed with different ball masses: $m \in\{0.1,1,2,3,4,5,6,7.26\} \mathrm{kg}$ (corresponding to black to light-gray lines) and fixed execution time $T=1 \mathrm{~s}$. The limits on $\phi$ are depicted with dashed red lines in Fig. 6(a1), (a2), (b1), and (b2) while the constraints on $\theta_{3,4}=\sigma_{1,2} \in[0,0.178] \mathrm{rad}$ are shown in Fig. 6(a3) and (b3). In addition, we set: $\theta_{1} \in[-1.05,3.05] \mathrm{rad}, \theta_{2} \in[2.27,0.44] \mathrm{rad}$, and $\dot{\theta}_{1,2} \in[-8,8] \mathrm{rad} / \mathrm{s}$ in these simulations as shown in Fig. 6(a4), (a5), (b4), and (b5). In all cases, the throwing motion is started from a vertical hanging configuration with minimum stiffness. Note that in Figs. 6(a1) and (b1) each solution (gray and black lines) strictly satisfies its own corresponding constraint (red dashed lines).

springs are given as

$$
\begin{gathered}
\mathbf{0} \preceq \boldsymbol{\sigma} \preceq \boldsymbol{\sigma}_{M},|\dot{\boldsymbol{\sigma}}| \preceq \dot{\boldsymbol{\sigma}}_{M} \\
\boldsymbol{\phi}_{m}(\boldsymbol{\sigma}) \preceq \boldsymbol{\phi} \preceq \boldsymbol{\phi}_{M}(\boldsymbol{\sigma})
\end{gathered}
$$

where the maximal limits are defined by: $\sigma_{M}=$ $0.178[1,1]^{T} \mathrm{rad}, \quad \dot{\boldsymbol{\sigma}}_{M}=0.541[1,1]^{T} \mathrm{rad} / \mathrm{s} \quad$ and $\quad \phi_{m}(\boldsymbol{\sigma})=$ $\left[\phi_{m 1}\left(\theta_{3}\right), \phi_{m 2}\left(\theta_{4}\right)\right]^{T}$, and $\boldsymbol{\phi}_{M}(\boldsymbol{\sigma})=\left[\phi_{M 1}\left(\theta_{3}\right), \phi_{M 2}\left(\theta_{4}\right)\right]^{T}$ are the state dependent constraint boundaries depicted in Fig. 5(c) (dashed red lines). The present formulation encodes these as hard constraints. Additional limits to be considered are the range/rate limits associated with the main motors [i.e., that modulate the set point of the virtual joint springs $\theta_{1,2}$, see Fig. 5(d)]. These constraints $\theta_{1,2 m} \leq \theta_{1,2} \leq \theta_{1,2 M},\left|\dot{\theta}_{1,2}\right| \leq \dot{\theta}_{1,2 M}$, where $\theta_{1,2 m}=[-1.05,-0.44]^{T} \mathrm{rad}, \quad \theta_{1,2 M}=[3.05,2.27]^{T} \mathrm{rad}, \quad$ and $\dot{\theta}_{1,2 M}=8[1,1]^{T} \mathrm{rad} / \mathrm{s}$ define the maximal ranges, are treated as soft constraints and embedded through penalty terms in the objective functional. This is one way to deal with constraint that cannot be embedded into the dynamics using the present formulation.

1) Simulation: A typical simulation result is shown in Fig. 6(a) corresponding to high bandwidth stiffness modulation and Fig. 6(b) corresponding to a low bandwidth one. In Fig. 6(a1) and (b1), one can see that the present formulation enforces the state inequality limits, as hard constraints. Fig. 6(a2) and (b2) shows that this is nontrivial since the boundaries of the constraints depend on stiffness modulation. Indeed, higher $\theta_{3}$ and $\theta_{4}$ in Fig. 6(a3) and (b3) result in stronger restriction on $\phi_{1}$ 


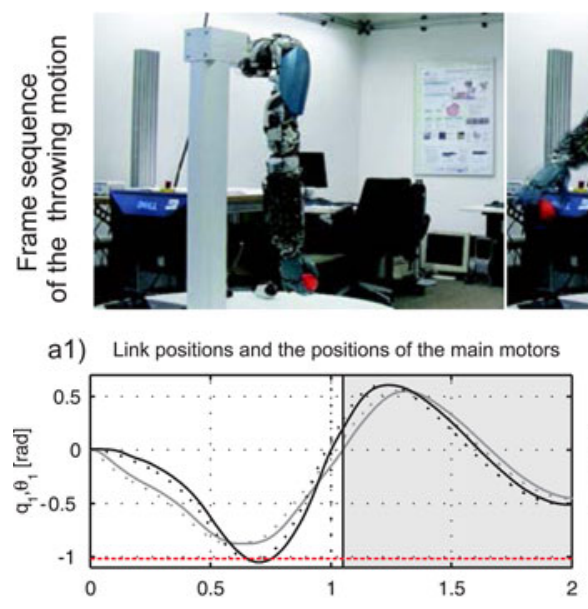

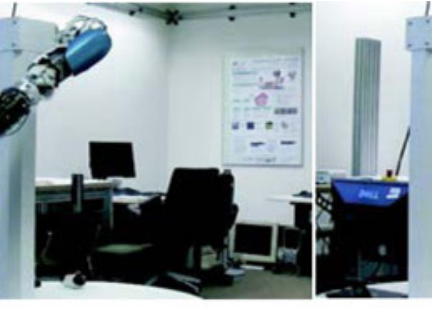

a2) Positions of the stiffness adjuster motors
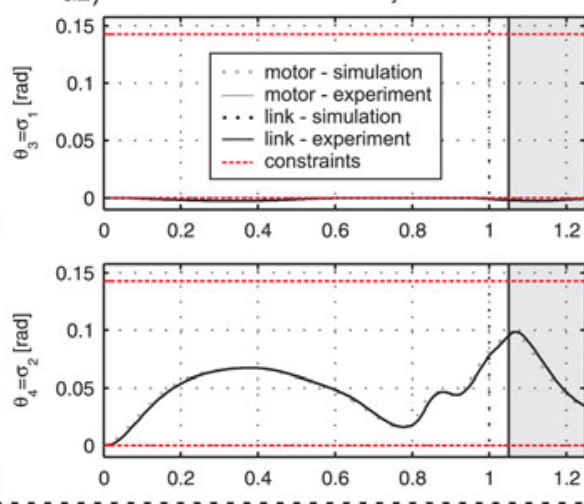

b2)
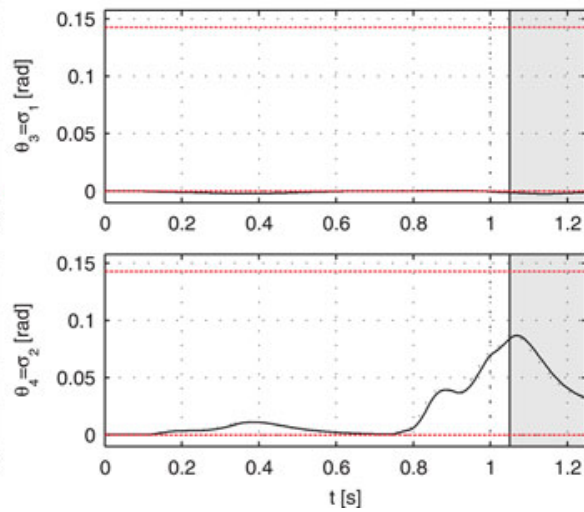
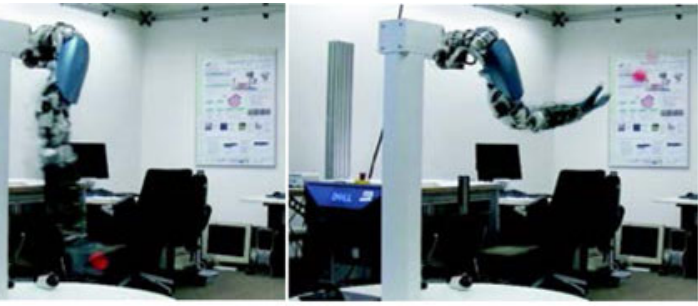

a3) Link velocities and the velocities of the main motors
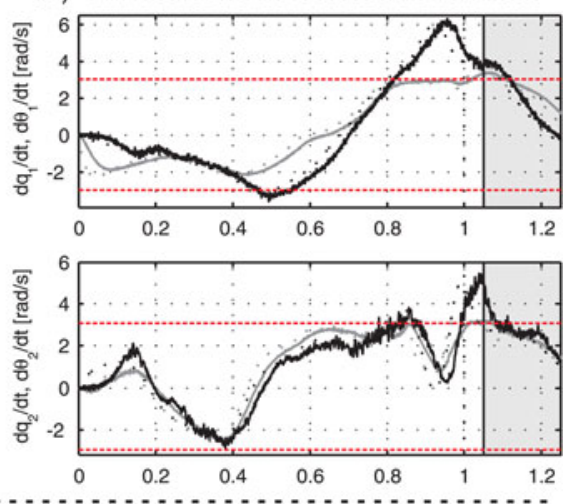

b3)
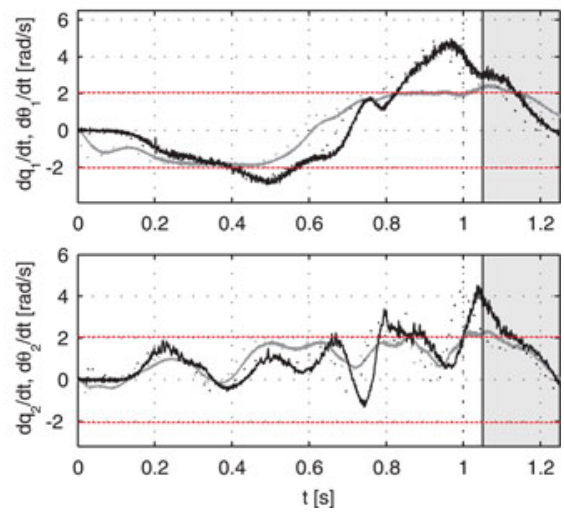

Fig. 7. Ball throwing using optimal variable stiffness control. (a1) and (b1) motor and link-side trajectories. (a2) and (b2) Stifness adjustment. (a3) and (b3) Motor and link-side velocities. Both of these experiments are performed using a fixed execution time $T=1 \mathrm{~s}$ and a ball weighting $m=0.06 \mathrm{~kg}$. For the first experiment (a) the motor velocity limits are set to $\dot{\theta}_{1,2} \in[-3,3] \mathrm{rad} / \mathrm{s}$, while for the second experiment (b) it was set to $\dot{\theta}_{1,2} \in[-2,2] \mathrm{rad} / \mathrm{s}$. The additional limits are defined by $\theta_{1} \in[-1.05,3.05] \mathrm{rad}, \theta_{2} \in[2.27,0.44] \mathrm{rad}, \theta_{3,4}=\sigma_{1,2} \in 0.8[0,0.178] \mathrm{rad}$, and $\boldsymbol{\alpha}=\operatorname{diag}[25,25,15,15] \mathrm{s}^{-1}$. The throwing motion is started from a vertical hanging configuration with no initial velocity and minimum stiffness. In these experiments stiffness modulation is restricted to the second joint. For further experiments on this system, see [40].

and $\phi_{2}$, respectively. In Fig. 6(a2) and (b2), one can also recognize a bang-bang like strategy (especially on the first joint). This strategy is planned under changing constraint boundaries and is smooth due to the bandwidth limitations of the actuators. Fig. 6(a4), (a5), (b4), and (b5) shows that neither of the constraints corresponding to the positions and velocities of the main motors (used to modulate the set-point of the virtual joint springs) are violated. This result demonstrates the applicability of the present optimal control formulation under nonlinear state inequality constraints that are often present as actuation constraints on robotic systems.

2) Experiment: Using the proposed framework, we computed and implemented one set of optimal motor trajectories that correspond to high bandwidth stiffness modulation realizing ball-throwing on the DLR-HASy. In Fig. 7, one can observe a good match between the simulated and the real link- side motions [i.e., $\mathbf{q}(t)$ ]. This is partly because of an accurate system model, but also because the proposed optimization algorithm plans motor trajectories [i.e., $\boldsymbol{\theta}(t)$ ] that respect all physical constraints and as such can be precisely tracked on this complex system. In addition, and similar to the simulations in Fig. 6, we can see a characteristic counter movement action, initial back swing before rapid forward acceleration [see Fig. 6(a) and (b) and the multimedia material], and a similar stiffness modulation pattern despite the difference in dynamics [see Fig. 6(a3)] and constraints [see Fig. 7(a2) and (b2)]. Moreover, we also recognize the characteristic sequential velocity peaks, first on the proximal link and then on the distal link in Fig. 7(a3) and (b3). These peaks are associated with the sequential (proximal-to-distal) motions of body segments often reported in human studies of striking and throwing skills [58]. 


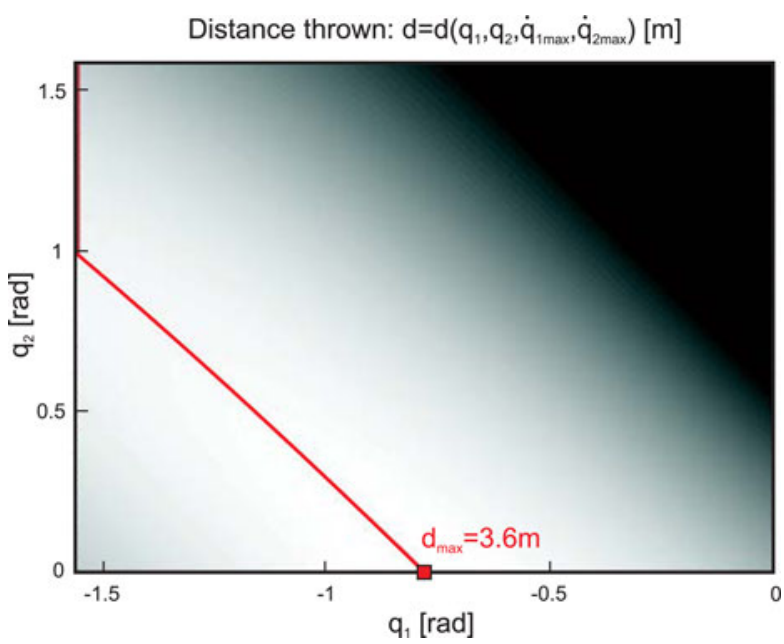

Fig. 8. Best throwing performance of a rigid robot as a function of the release configuration and under a given velocity limit: $\dot{\theta}_{1,2} \in[-3,3] \mathrm{rad} / \mathrm{s}$, i.e., $\dot{\mathbf{q}}_{\max }=$ $[3,3]^{T} \mathrm{rad} / \mathrm{s}$.

3) Stiffness Modulation: By means of stiffness modulation, we observe a characteristic decrease followed by a rapid increase of the stiffness just before the ball release $(t \approx 1 \mathrm{~s})$ [see Figs. 6(a3), and 7(a2), (b2)]. This stiffness modulation strategy bears clear physical interpretation. Indeed, decreased stiffness, near to the end of the movement, enables the link to maximally depart from its equilibrium configuration. This then makes it possible to accelerate the arm by increasing the stiffness just before ball release. This mechanism is predicted to be optimal on the present robotic system. Whether a similar mechanism is employed during human throwing and striking motions has yet to be investigated.

It is important to recognize that a stiffness modulation strategy that has been shown in Figs. 6(a3),7(a2), and (b2) is not possible in the case of fixed stiffness control [18] or when the bandwidth of the actuators associated with stiffness modulation is low [see Fig. 6(b3)]. This leads to lower performance (i.e., shorter distance thrown) [see Fig. 6(b)].

4) Variable Stiffness Versus Rigid Actuation: Finally, we investigate how the performance of the optimally controlled compliantly actuated robot (i.e., DLR HASy) compares with the best performance of the corresponding rigid robot (where $\dot{\mathbf{q}}=\left[\dot{\theta}_{1}, \dot{\theta}_{2}\right]^{T}$ ). To ensure a common ground for this comparison, we consider both systems to be subject to the same motor velocity limits. i.e., $\dot{\theta}_{1} \in[-3,3] \mathrm{rad} / \mathrm{s}$ and $\dot{\theta}_{2} \in[-3,3] \mathrm{rad} / \mathrm{s}$, as well as assume no-torque limit on the rigid robot. ${ }^{17}$ By comparing the link-side velocities, it becomes immediately clear that the same velocity peaks shown in Figs. 7(a3) and (b3) could not have been achieved with a rigid robot. When reflected to the task performance, we found by kinematic calculation that while the rigid robot could, under no effort limitation, throw a ball $d=3.6 \mathrm{~m}$ (see Fig. 8), the VS joints allowed the DLR HASy

\footnotetext{
${ }^{17}$ This latter assumption makes the restrictions on the rigid robot weaker compared with those that apply on the DLR HASy, where the elastic joint torques are limited, both in range and bandwidth.
}

to throw a ball $d \approx 5 \mathrm{~m}$. (For the corresponding experimental video, see the multimedia attachment).

This comparison shows the ability of the present optimal control framework to exploit the rigid body dynamics and the capacity of the compliant actuators to achieve better task performance. Moreover, it demonstrates the utility of optimal impedance control and the advantage of compliant design for the next generation of robotic systems.

\section{CONCLUSION}

In this paper, we provide an optimal-control formulation for compliantly actuated robots subject to actuation constraints. Instead of employing problem specific analytical derivations or using sophisticated numerical algorithms to treat the inequality constraints inevitably present on compliantly actuated robots, this formulation employs canonical box-constraints on control inputs to explicitly embed the actuation constraints into the dynamics. In practice, these actuation constraints can be range limits on the motor positions, bandwidth limitations due to the motor dynamics, as well as deformation limits on the elastic elements inherent to many SEA and VSA designs. In this paper, we: 1) illustrate the impact of these limits on the performance and the control strategy during optimal variable stiffness control; and 2) demonstrate the viability of the proposed formulation on two conceptually different variable-stiffness system that perform a dynamic task in simulations and experiments. The proposed framework may be further developed by integration of: 1) a mechanism for active feedback control, to deal with unforeseen disturbances and environmental uncertainties; and 2) a framework for online dynamics adaptation, to deal with systematic changes in the dynamics.

Finally, we note that this investigation has been partly motivated by the latest hardware developments in anthropomorphic robots, exoskeletons, and prosthetic devices, where compliance is deliberately introduced into the system. In that regard, this study demonstrates the utility and viability of the proposed optimal control framework and provide some valuable design guidelines for the next generation of robotic devices, where compliant actuation and variable impedance control is likely to play a dominant role.

\section{APPENDIX \\ APPROACHES TO CONSTRAINED OPTIMIZATION}

Here, we consider several alternative approaches that can be used to solve (43)-(45). The purpose of this section is to highlight the benefits of different methods and to point out the practical difficulties related to the treatment of complex (i.e., high-dimensional, nonlinear, and constrained) optimal control problems. For a more elaborate exposition on this matter, the reader may refer to [45].

\section{A. Indirect Methods}

In an indirect approach, one attempts to find a solution that satisfies the first-order optimality conditions to the original constrained functional minimization (43)-(45). For this purpose: 
1) the first-order optimality conditions are derived using Pontryagin's maximum principle [59]; 2) these conditions are then represented with a multipoint boundary value problem (MPBVP) [44]; and finally 3) the obtained MPBVP is solved using a multiple-shooting method (see [60]). The MPBVP generally contains the state equations, adjoint equations, transversality conditions, and differential equations that locate the entry and exit points for every state and control constrained subarcs and the associated jump conditions. ${ }^{18}$

There are notable differences in this representation and the corresponding solution, depending on whether state or only control constraints are considered. This is because state inequalities lead to piecewise defined adjoint equations and discontinuous adjoint functions. This complicates the treatment of state constrained formulations, partly because the discontinuities may lead to sensitivity/accuracy issues during numerical integration, but also because the number of functions that represent the piecewise defined adjoint equations has to be guessed. Both of these issues can be circumvented using the present formulation that is subject to control constraints only.

Even if only control constraints are present, however, application of multiple-shooting methods generally requires the user to guess the missing initial data to integrate the adjoint equations, and to find the structure of the control constraint subarcs. This imposes strong restrictions, namely to make the numerical algorithm converge, the user must guess the details of the optimal solution. [61].

\section{B. Direct Methods}

In direct methods, one employs control and/or state parametrization to transform the original optimal control problem, e.g., (43)-(45), to a nonlinear programming problem (NLP) [62]. Instead of finding a solution to the first-order optimality conditions, the original infinite dimensional constrained functional minimization is directly converted to a finite dimensional function minimization that is easier to solve numerically. Indeed, such a function minimization can be solved using sophisticated NLP methods, e.g., using sequential quadratic programming [63].

There are numerous reasons that make this formulation attractive. One of these is that, direct methods do not require the optimality conditions to be analytically derived (that is often nontrivial and cumbersome for high-dimensional constrained problems). Moreover, unlike multiple shooting methods, nonlinear programing is robust to initializations. Notably, for constrained problems, nonlinear programming can be applied without a prior specification of the sequence and number of constrained subarcs. This is because the entry and exit points on these sub-arcs are automatically identified by standard active set algorithms [45].

On the other hand, however, control and state parameterization generally leads to a large-scale NLP. Due to this reason, exploiting the sparsity pattern of such an NLP is essential for computationally tractable implementation. Even if the

\footnotetext{
${ }^{18}$ Erdmann-Weierstraß corner conditions [61].
}

computation is optimized in this way, there is a natural tradeoff between computational tractability and the accuracy of the solution this approach provides. To circumvent possible accuracy issues, merging direct and indirect methods has been suggested [64]. Alternatively, successive approximation methods may also be employed.

\section{Successive Approximation Methods}

The idea behind these methods is to derive a subproblem that can be iteratively solved to improve the nominal control trajectory, initially provided by the user. Examples of these approaches are: differential dynamic programming (DDP) [65], the iterative linear quadratic regulator/Gaussian (iLQR/G) approach [53], as well as many other first- and second-order methods that are derived from Bellman's dynamic programming or by using variational calculus [66]. Compared with direct methods, these iterative approaches provide more precise solutions while compared with indirect methods, these approaches are robust against initializations. In this light, many of the issues discussed previously (e.g., computational efficiency, sensitivity to initialization, accuracy of the solution) could be addressed using methods for successive approximation. Authors in [67] provide a unified view of such approximation methods in the language of KL divergence minimization. While these methods are primarily derived to solve unconstrained problems, there are also many alternatives when optimizing under state and control constraints [68].

In the present context, the state constraints are explicitly embedded in the dynamics, such that any method allowing boxconstraints on controls would suffice. One of these methods is provided with the iLQR/G approach [53] that is applicable to nonlinear problems and remains efficient for high-dimensional systems. This approach is used in Section VII.

\section{REFERENCES}

[1] M. Grebenstein, A. Albu-Schäffer, T. Bahls, M. Chalon, O. Eiberger, W. Friedl, R. Gruber, U. Hagn, R. Haslinger, H. Höppner, S. Jörg, M. Nickl, A. Nothhelfer, F. Petit, B. Pleintinger, J. Reil, N. Seitz, T. Wimböck, S. Wolf, T. Wüsthoff, and G. Hirzinger, "The DLR hand arm system," in Proc. IEEE Int. Conf. Robot. Autom., Shanghai, China, 2011, pp. 3175-3182.

[2] C. E. English, "Implementation of variable joint stiffness through antagonistic actuation using rolamite springs," Mech. Mach. Theory, vol. 341, pp. 27-40, 1999.

[3] C. E. English and D. Russell, "Mechanics and stiffness limitations of a variable stiffness actuator for use in prosthetic limbs," Mech. Mach. Theory, vol. 341, pp. 7-25, 1999.

[4] K. Koganezawa, Y. Watanabe, and N. Shimizu, "Antagonistic muscle-like actuator and its application to multi-DOF forearm prosthesis," Adv. Robot., vol. 12, no. 7-8, pp. 771-789, 1999.

[5] G. Tonietti, R. Schiavi, and A. Bicchi, "Design and control of a variable stiffness actuator for safe and fast physical human/robot interaction," in Proc. IEEE Int. Conf. Robot. Autom., Barcelona, Spain, 2005, pp. 526531.

[6] S. A. Migliore, E. A. Brown, and S. P. DeWeerth, "Novel nonlinear elastic actuators for passively controlling robotic joint compliance," J. Mech. Design, vol. 129, no. 4, pp. 406-412, 2007.

[7] S. Wolf and G. Hirzinger, "A new variable stiffness design: Matching requirements of the next robot generation," in Proc. IEEE Int. Conf. Robot. Autom., Pasadena, CA, USA, 2008, pp. 1741-1746. 
[8] R. van Ham, T. Sugar, B. Vanderborght, K. Hollander, and D. Lefeber, "Compliant actuator designs," IEEE Robot. Autom. Mag., vol. 16, no. 3, pp. 81-94, Sep. 2009.

[9] A. Radulescu, M. Howard, D. J. Braun, and S. Vijayakumar, "Exploiting variable physical damping in rapid movement tasks," in Proc. IEEE/ASME Int. Conf. Adv. Intell. Mechatron., Kaohsiung, Taiwan, Jul. 2012, pp. 141148.

[10] B. Siciliano and O. Khatib, Handbook of Robotics. New York, NY, USA: Springer-Verlag, 2008.

[11] N. Hogan, "Impedance control: An approach to manipulation," ASME J. Dyn. Syst., Meas. Control, vol. 107, pp. 1-24, 1985.

[12] J. W. Hurst, J. Chestnutt, and A. A. Rizzi, "The actuator with mechanically adjustable series compliance," IEEE Trans. Robot., vol. 26, no. 4, pp. 597606, Aug. 2010.

[13] B. Vanderborght, B. Verrelst, R. V. Ham, M. V. Damme, D. Lefeber, B. M. Y. Duran, and P. Beyl, "Exploiting natural dynamics to reduce energy consumption by controlling the compliance of soft actuators," Int. J. Robot. Res., vol. 25, no. 4, pp. 343-358, 2006.

[14] A. Bicchi and G. Tonietti, "Fast and soft arm tactics: Dealing with the safety-performance trade-off in robot arms design and control," IEEE Robot. Autom. Mag., vol. 11, no. 2, pp. 22-33, Jun. 2004.

[15] D. Mitrovic, S. Klanke, and S. Vijayakumar, "Learning impedance contro of antagonistic systems based on stochastic optimization principles," Int. J. Robot. Res., vol. 30, no. 2, pp. 1-18, 2011 .

[16] D. J. Braun, M. Howard, and S. Vijayakumar, "Exploiting variable stiffness in explosive movement tasks," presented at the Robot.: Sci. Syst., Los Angeles, CA, USA, Jun./Jul. 2011.

[17] S. Haddadin, M. Weis, S. Wolf, and A. Albu-Schäffer, "Optimal control for maximizing link velocity of robotic variable stiffness joints," presented at the IFAC World Congr., Milano, Italy, Aug./Sep. 2011.

[18] D. J. Braun, M. Howard, and S. Vijayakumar, "Optimal variable stiffness control: Formulation and application to explosive movement tasks," Auton. Robots, vol. 33, no. 3, pp. 237-253, 2012.

[19] M. Spong, "Modeling and control of elastic joint robots," J. Dyn. Syst., Meas., Control, vol. 109, no. 4, pp. 310-319, 1987.

[20] P. Tomei, "A simple PD controller for robots with elastic joints," IEEE Trans. Automat. Control, vol. 36, no. 10, pp. 1208-1213, Oct. 1991.

[21] G. Pratt and M. Williamson, "Series elastic actuators," in Proc. IEEE/RSJ Int. Conf. Intell. Robots Syst., Pittsburgh, PA, USA, 1995, vol. 1, pp. 399406.

[22] A. DeLuca, F. Flacco, A. Bicchi, and R. Schiavi, "Nonlinear decoupled motion-stiffness control and collision detection/reaction for the VSA-II variable stiffness device," in Proc. IEEE/RSJ Int. Conf. Intell. Robots Syst., St. Louis, MO, USA, Oct. 2009, pp. 5487-5494.

[23] A. Albu-Schäffer, S. Wolf, O. Eiberger, S. Haddadin, F. Petit, and M. Chalon, "Dynamic modelling and control of variable stiffness actuators," in Proc. IEEE Int. Conf. Robot. Autom., Anchorage, AK, USA, May 2010, pp. 2155-2162.

[24] D. J. Braun, F. Petit, F. Huber, S. Haddadin, P. Smagt, A. Albu-Schäffer, and S. Vijayakumar, "Optimal torque and stiffness control in compliantly actuated robots," in Proc. IEEE/RSJ Int. Conf. Intell. Robots Syst., Vilamoura, Portugal, Oct. 2012, pp. 2801-2808.

[25] B. Brogliato, R. Ortega, and R. Lozano, "Global tracking controller for flexible-joint manipulators: A comparative study," Automatica, vol. 31, no. 7, pp. 941-956, 1995.

[26] M. W. Spong, K. Khorasani, and P. V. Kokotović, "An integral manifold approach to the feedback control of flexible joint robots," IEEE J. Robot. Autom., vol. RA-3, no. 4, pp. 291-300, Aug. 1987.

[27] A. DeLuca, "Feedforward/feedback laws for the control of flexible robots," in Proc. IEEE Int. Conf. Robot. Autom., San Francisco, CA, USA, Apr. 2000, pp. 233-240.

[28] S. Nicosia and P. Tomei, "A method to design adaptive controllers for flexible joint robots," in Proc. IEEE Int. Conf. Robot. Autom., May 1992 vol. 1, pp. 701-706.

[29] A. Albu-Schäffer and G. Hirzinger, "A globally stable state-feedback controller for flexible joint robots," J. Adv. Robot., vol. 15, no. 8, pp. 799814,2001

[30] C. Ott, A. Albu-Schäffer, A. Kugi, and G. Hirzinger, "On the passivitybased impedance control of flexible joint robots," IEEE Trans. Robot., vol. 24, no. 2, pp. 416-429, Apr. 2008.

[31] G. Palli, C. Melchiorri, T. Wimbock, M. Grebenstein, and G. Hirzinger, "Feedback linearization and simultaneous stiffness-position control of robots with antagonistic actuated joints," in Proc. IEEE Int. Conf. Robot. Autom., Roma, Italy, May 2007, pp. 4367-4372.
[32] G. Palli, C. Melchiorri, and A. D. Luca, "On the feedback linearization of robots with variable joint stiffness," in Proc. IEEE Int. Conf. Robot. Autom., Pasadena, CA, USA, May 2008, pp. 1753-1759.

[33] L. Ljung, System Identification: Theory for the User. Upper Saddle River, NJ, USA: Prentice-Hall, 1999.

[34] S. Vijayakumar, A. D'Souza, and S. Schaal, "Incremental online learning in high dimensions," Neural Comput., vol. 17, pp. 2602-2634, 2005

[35] D. Mitrovic, S. Klanke, and S. Vijayakumar, "Adaptive optimal feedback control with learned internal dynamics models," in From Motor Learning to Interaction Learning in Robots. New York, NY, USA: SpringerVerlag, 2010, vol. 264, pp. 65-84.

[36] K. J. Salisbury, "Active stiffness control of a manipulator in cartesian coordinates," in Proc. 19th IEEE Conf. Decis. Control, Dec. 1980, vol. 19 pp. 95-100.

[37] N. Hogan, "Impedance control of industrial robots," Robot. Comput.Integr. Manuf., vol. 1, no. 1, pp. 97-113, 1984.

[38] M. Uemura and S. Kawamura, "Resonance-based motion control method for multi-joint robot through combining stiffness adaptation and iterative learning control," in Proc. IEEE Int. Conf. Robot. Autom., Kobe, Japan, 2009 , pp. 1543-1548.

[39] M. Garabini, A. Passaglia, F. A. W. Belo, P. Salaris, and A. Bicchi, "Optimality principles in variable stiffness control: The VSA hammer," in Proc. IEEE/RSJ Int. Conf. Intell. Robots Syst., San Francisco, CA, USA, Sep. 2011, pp. 3770-3775.

[40] S. Haddadin, F. Huber, and A. Albu-Schäffer, "Optimal control for exploiting the natural dynamics of variable stiffness robots," in Proc. IEEE Int. Conf. Robot. Autom., St Paul, MN, USA, 2012, pp. 3347-3354.

[41] J. Nakanishi and S. Vijayakumar, "Exploiting passive dynamics with variable stiffness actuation in robot brachiation," presented at Robotics: Science and Systems, Sydney, Australia, 2012.

[42] J. E. Bobrow, S. Dubowsky, and J. S. Gibson, "Time-optimal control of robotic manipulators along specified paths," Int. J. Robot. Res., vol. 4, no. 3, pp. 3-17, 1985.

[43] D. S. Bernstein and A. N. Michel, "A chronological bibliography on saturating actuators," Int. J. Robust Nonlinear Control, vol. 5, pp. 375-380, 1995.

[44] H. J. Pesch, "A practical guide to the solution of real-life optimal control problems," Control Cybern., vol. 23, no. 1, pp. 7-60, 1994.

[45] J. T. Betts, "Survey of numerical methods for trajectory optimization," AIAA J. Guid., Control Dyn., vol. 21, no. 2, pp. 193-207, 1998.

[46] A. DeLuca, B. Siciliano, and L. Zollo, "PD control with on-line gravity compensation for robots with elastic joints: Theory and experiments,' Automatica, vol. 41, pp. 1809-1819, 2005.

[47] H. Goldstein, Classical Mechanics. Reading, MA, USA: AddisonWesley, 1980.

[48] G. Golub and C. V. Loan, Matrix Computations, 3rd ed. Baltimore, MD, USA: Johns Hopkins Univ. Press, 1996.

[49] W. Rudin, Principles of Mathematical Analisys, 3rd ed. New York, NY, USA: McGraw-Hill, 1953.

[50] C. T. Kelley, Solving Nonlinear Equations with Newton's Method. Philadelphia, PA, USA: SIAM, 2003.

[51] M. Goldfarb and T. Sirithanapipat, "The effect of actuator saturation on the performance of PD-controlled servo systems," Mechatronics, vol. 9 pp. 497-511, 1999 .

[52] W. L. Nelson, "Physical principles for economies of skilled movements," Biol. Cybern., vol. 46, no. 2, pp. 135-147, 1983

[53] W. Li and E. Todorov, "Iterative linearization methods for approximately optimal control and estimation of non-linear stochastic system," Int. J. Control, vol. 80, no. 9, pp. 1439-1453, 2007.

[54] R. van Ham, B. Vanderborght, M. V. Damme, B. Verrelst, and D. Lefeber, "MACCEPA, the mechanically adjustable compliance and controllable equilibrium position actuator: Design and implementation in a biped robot," Robot. Auton. Syst., vol. 55, no. 10, pp. 761-768, 2007.

[55] N. Hogan, "Adaptive control of mechanical impedance by coactivation of antagonist muscles," IEEE Trans. Automat. Control, vol. AC-29, no. 8, pp. 681-690, Aug. 1984

[56] J. M. Winters and L. Stark, "Analysis of fundamental human movement patterns through the use of in-depth antagonistic muscle models," IEEE Trans. Biomed. Eng., vol. 32, no. 10, pp. 826-839, Oct. 1985.

[57] M. Howard, D. J. Braun, and S. Vijayakumar, "Transferring human impedance behaviour to heterogeneous variable impedance actuators," IEEE Trans. Robot., vol. 29, no. 4, 2013, doi: 10.1109/TRO.2013.2256311 
[58] C. Putnam, "Sequential motions of body segments in striking and throwing skills: Descriptions and explanations," J. Biomech., vol. 26, no. 1, pp. 125135, 1993.

[59] L. S. Pontryagin, V. G. Boltyanskii, R. V. Gamkrelidze, and E. F. Mishchenko, The Mathematical Theory of Optimal Processes. New York, NY, USA: Wiley, 1962.

[60] U. Ascher, R. Mattheij, and R. D. Russell, Numerical Solution of Boundary Value Problems for Differential Equations. Philadelphia, PA, USA: SIAM, 1988.

[61] A. E. Bryson and Y. C. Ho, Applied Optimal Control. Bristol, PA, USA: Wiley, 1975.

[62] C. R. Hargraves and S. W. Paris, "Direct trajectory optimization using nonlinear programming and collocation," J. Guid. Control Dyn., vol. 10, pp. 338-342, 1987.

[63] P. E. Gill, W. Murray, M. A. Saunders, and M. H. Wright, User's guide for NPSOL (ver. 4) Report SOL 86-2, Dept. Oper. Res., Stanford Univ., Stanford, CA, USA, 1986.

[64] O. von Stryk and R. Bulirsch, "Direct and indirect methods for trajectory optimisation," Ann. Oper. Res., vol. 37, pp. 357-373, 1992.

[65] D. H. Jacobson and D. Q. Mayne, Differential Dynamic Programming. New York, NY, USA: Elsevier, 1970.

[66] D. H. Jacobson, "Second-order and second-variation methods for determining optimal control: A comparative study using differential dynamic programming," Int. J. Control, vol. 7, no. 2, pp. 175-196, 1968.

[67] K. Rawlik, M. Toussaint, and S. Vijayakumar, "On stochastic optimal control and reinforcement learning by approximate inference," presented at Robot.: Sci. Syst., Sydney, Australia, 2012.

[68] S. J. Yakowitz, "The stagewise Kuhn-Tucker condition and differential dynamic programming," IEEE Trans. Automat. Control, vol. AC-31, no. 1, pp. 25-30, Jan. 1986.

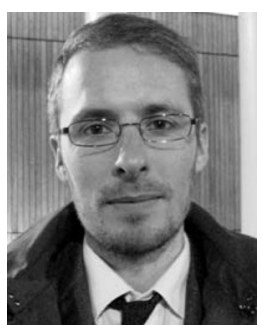

actuation
David J. Braun (M'09) received the Ph.D. degree in mechanical engineering from Vanderbilt University, Nashville, TN, USA, in 2009.

Following that, he was affiliated with the Center for Intelligent Mechatronics at Vanderbilt and conducted research at the Institute of Robotics and Mechatronics, German Aerospace Center. $\mathrm{He}$ is currently a Postdoctoral Research Fellow with the University of Edinburgh, Edinburgh, U.K. His research interests include system dynamics, optimal control, impedance control, and variable impedance

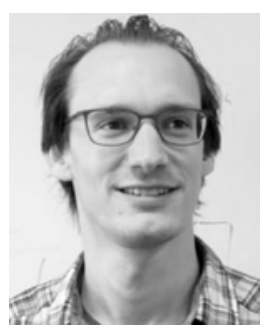

Florian Petit received the Dipl.-Ing. degree in electrical engineering from the Technical University of Munich, Munich, Germany, in 2008.

He joined the Institute of Robotics and Mechatronics, German Aerospace Center, Wessling, Germany, in 2009. He has also been a member of the Sensory-Motor Systems Laboratory, Swiss Federal Institute of Technology Zurich, Zurich, Switzerland, since 2012. His research interests include variable impedance robots and control, impedance and force control, and nonlinear systems and control.

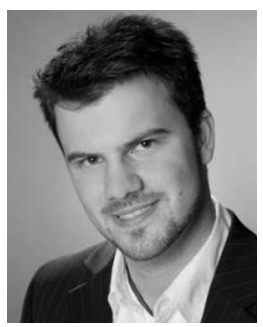

Felix Huber received the Dipl.-Ing. degree in mechatronics from Johannes Kepler University, Linz, Austria, in 2012 .

$\mathrm{He}$ is currently a Research Engineer with the Institute of Robotics and Mechatronics, German Aerospace Center, Wessling, Germany. His research interests include robot modeling and control and optimization

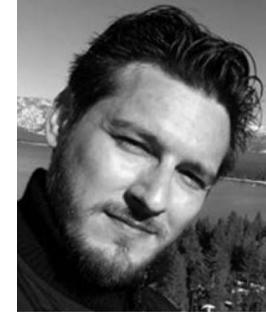

Sami Haddadin (M'10) received the Ph.D. degree (summa cum laude) from RWTH Aachen, Aachen, Germany, in 2011.

$\mathrm{He}$ coordinates the Institute of Robotics and Mechatronics, German Aerospace Center's "Human Centered Robotics" Group and is a Lecturer with the Technical University of Munich, Wessling, Germany. He has published more than 65 papers/chapters in journals, books, and conferences. His research interests include physical human-robot interaction, real-time planning, control and learning, variable impedance actuator, optimal control, and safety.

Dr. Haddadin organized and was a PC member/editor of several workshops and conferences. He received six International Conference on Robotics and $\mathrm{Au}-$ tomation/IROS awards, the 2012 George Giralt Best Ph.D. Thesis Award, the IEEE TRANSACTIONS ON ROBOTICS 2011 Best Paper Award, and was a finalist for the Robotdalen Science Award in 2009. He was the Guest Editor of International Journal of Robotics Research.

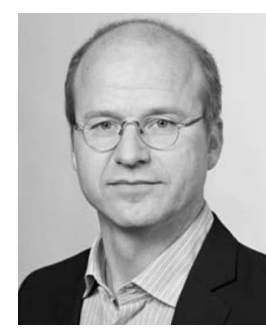

Patrick van der Smagt (M'12) received the Drs. degree from the Vrije Universiteit Amsterdam, Amsterdam, The Netherlands, in 1990 and the Ph.D. degree in computer science and mathematics from the University of Amsterdam in 1995

He is currently Professor for biomimetic robotics and machine learning with the Technical University of Munich, Munich, Germany. His research interests include machine learning, neural control of movement, robotics, and brain-machine interfaces.

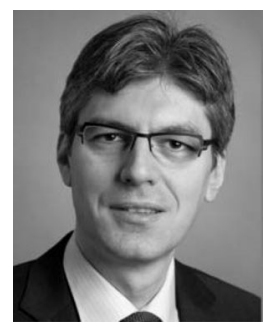

Alin Albu-Schäffer (M'93) received the Diploma degree in electrical engineering from the Technical University of Timisoara, Timisoara, Romania, in 1993 and the Ph.D. degree in control systems from the Technical University of Munich, Munich, Germany, in 2002.

Since 2012, he has been Head of the Institute of Robotics and Mechatronics, German Aerospace Center, Wessling, Germany, which he joined in 1995. His research interests include robot design, modeling, and control, nonlinear control, flexible joint and variable compliance robots, impedance and force control, and physical human-robo interaction.

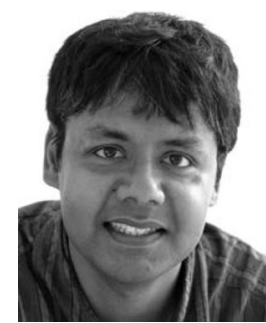

Sethu Vijayakumar received the Ph.D. degree in computer science and engineering from the Tokyo Institute of Technology, Tokyo, Japan, in 1998.

$\mathrm{He}$ is Professor of Robotics and a Director with the Institute of Perception, Action, and Behavior, School of Informatics, University of Edinburgh, U.K., where he holds the Royal Academy of Engineering Microsoft Research Chair in Learning Robotics. He also has additional appointments as an Adjunct Faculty with the University of Southern California, Los Angeles, CA, USA and a Visiting Research Scientist with the RIKEN Brain Science Institute, Tokyo. His research interests include statistical machine learning, robotics, planning and optimization in autonomous systems to human motor control, and optimality.

Dr. Vijayakumar is a Fellow of the Royal Society of Edinburgh. 